\title{
UNCERTAINTY ON RADIATION DOSES ESTIMATED BY BIOLOGICAL AND RETROSPECTIVE PHYSICAL METHODS
}

Elizabeth A. Ainsbury ${ }^{1, *}$, Daniel Samaga ${ }^{2}$, Sara Della Monaca ${ }^{3}$, Maurizio Marrale ${ }^{4}$, Celine Bassinet ${ }^{5}$, Christopher $^{3}$ I. Burbidge ${ }^{6}$, Virgilio Correcher ${ }^{7}$, Michael Discher ${ }^{8}$, Jon Eakins ${ }^{1}$, Paola Fattibene ${ }^{3}$, İnci Güçlï ${ }^{9}$, Eva Lund ${ }^{10}$, Nadica Maltar-Strmečki ${ }^{11}$, Steve McKeever ${ }^{12}$, Christopher L. Rääf ${ }^{13}$, Sergey Sholom ${ }^{12}$, Ivan Veronese ${ }^{14}$, Albrecht Wieser $^{15}$, Clemens Woda ${ }^{15}$, Manuel Higueras ${ }^{16}$ and Francois Trompier ${ }^{5}$.

${ }^{1}$ Public Health England, Centre for Radiation, Chemical and Environmental Hazards, Chilton, Didcot, Oxford OX11 ORQ, UK.

${ }^{2}$ Bundesamt für Strahlenschutz, Ingolstaedter Landstr. 1, 85764 Oberschleissheim, Germany.

${ }^{3}$ Istituto Superiore di Sanità, Viale Regina Elena 299, 00161, Rome, Italy.

${ }^{4}$ Department of Physics and Chemistry and AteN Center, University of Palermo, Viale delle Scienze Edificio 18, 90128, Palermo, Italy.

${ }^{5}$ Institut de radioprotection et de sûreté nucléaire, BP 17 - 92262 Fontenay-aux-Roses Cedex 31, Avenue de la Division Leclerc 92260 Fontenay-aux-Roses, Paris, France.

${ }^{6}$ Environmental Protection Agency, Office of Radiological Protection, 3 Clonskeagh Square, Clonskeagh Road, Dublin 14, Ireland.

${ }^{7}$ Centro de Investigaciones Energéticas, Medioambientales y Tecnológicas (CIEMAT), Centro de la Moncloa, Complutense, 40, 28040, Madrid, Spain.

${ }^{8}$ Universitat Salzburg, Department of Geography and Geology, Hellbrunnerstraße 34, 5020 Salzburg, Austria.

${ }^{9}$ Turkish Atomic Energy Authority, Mustafa Kemal Mahallesi, Dumlupınar Bulvarı, No: 192, 06510, Çankaya Ankara, Turkey.

${ }^{10}$ Department of Medical and Health Sciences, Linköping University, SE-581 85 Linköping, Sweden.

${ }^{11}$ Ruđer Bošković Institute, Division of Physical Chemistry, Laboratory for Magnetic Resonances, Bijenička cesta 54,10000 Zagreb, Croatia.

${ }^{12}$ Oklahoma State University, 145 Physical Sciences, Campus, Stillwater, OK 74078, USA.

${ }^{13}$ Medicinsk strålningsfysik, Institutionen för Translationell Medicin, Lunds universitet, Skånes universitetssjukhus SUS, SE-205 02, Malmö, Sweden.

${ }^{14}$ Università degli Studi di Milano, Department of Physics and National Institute of Nuclear Physics (INFN), Section of Milan, Via Celoria 16, 20133 - Milano (MI), Italy.

${ }^{15}$ Helmholtz Zentrum München, Deutsches Forschungszentrum für Gesundheit und Umwelt, Institute of Radiation Protection, Ingolstädter Landstraße 1, D-85764 Neuherberg, Germany.

${ }^{16}$ Basque Center for Applied Mathematics, Alameda de Mazarredo 14, E-48009 Bilbao, Basque Country, Spain.

Received month date year, amended month date year, accepted month date year

*Corresponding author: Tel: 00441235 825105; Fax: 00441235 833891; Email: liz.ainsbury@phe.gov.uk 


\section{UNCERTAINTY ON RADIATION DOSES ESTIMATED BY BIOLOGICAL AND RETROSPECTIVE PHYSICAL METHODS}

Elizabeth A. Ainsbury, Daniel Samaga, Sara Della Monaca, Maurizio Marrale, Celine Bassinet, Christopher I. Burbidge, Virgilio Correcher, Michael Discher, Jonathan Eakins, Paola Fattibene, İnci Güçlü, Eva Lund, Nadica Maltar-Strmečki, Steve McKeever, Christopher L. Rääf, Sergey Sholom, Ivan Veronese, Albrecht Wieser, Clemens Woda and Francois Trompier.

Biological and physical retrospective dosimetry are recognised as key techniques to provide individual estimates of dose following accidental or unexpected exposures to ionising radiation. Whilst there has been a relatively large amount of recent development in the biological and physical procedures, statistical development of uncertainty analysis techniques has in many areas failed to keep pace. The aim of this paper is to review the current state of the art in uncertainty analysis techniques across the EURADOS Working Group 10 - Retrospective dosimetry partners, to give concrete examples of implementation of the techniques recommended in the international standards, and to further promote the use of Monte Carlo techniques to support characterization of uncertainties. It is concluded that sufficient techniques are available and in use by most laboratories for acute, whole body exposures to highly penetrating radiation, but that further work will be required to ensure that the standards are adequately applied for the more complex exposure scenarios.

\section{INTRODUCTION}

In Europe today, and indeed the whole world, the current state of the art of retrospective radiation dosimetry incorporates a number of key biological and physical retrospective techniques. Amongst the biological methods, the dicentric chromosome assay (DCA), which relies on the relationship between the frequency of dicentric chromosomes in peripheral blood lymphocytes and the absorbed dose ("dose") of exposed persons, is recognised to be the most welldeveloped of these $\mathrm{e}^{1,2}$. The cytokinesis-blocked micronucleus (CBMN) assay is also regarded as an important tool for biological assessment of radiation dose and is growing in importance ${ }^{3}$. For longer-term retrospective dosimetry, fluorescence in situ hybridization (FISH) staining of translocations can be used $^{4}$. In addition, new techniques for premature chromosome condensation and scoring offer alternative methodologies $^{5}$ and the $\gamma$-H2AX assay allows direct measurement of double-strand breaks (DSB), which are caused almost exclusively by ionising radiation, in a human cell ${ }^{6}$. For physical dosimetry ${ }^{7}$, the key techniques are electron paramagnetic resonance (EPR) with bone ${ }^{8}$, teeth ${ }^{9}$, nails ${ }^{10,11,12}$ and glass ${ }^{13}$ and optically or thermally stimulated luminescence (OSL/TL), chiefly of electronic components in mobile phones ${ }^{14}$. A large number of studies have demonstrated the need for a wider range of techniques, both separately and in collaboration, in order that dosimetry can be carried out in several potential exposure scenarios ${ }^{15,16}$. Most importantly, the operational assays have recently been amalgamated into fully functional emergency response plans through the network: Realising the European network of biodosimetry, RENEB ${ }^{16,17}$.

The European Radiation Dosimetry Group (EURADOS) was set up to advance the scientific understanding and the technical development of the dosimetry of ionising radiation by the stimulation of collaboration between laboratories in Europe. As part of the network, EURADOS Working Group 10 (WG10) was formed, with the objective of establishing a network of European (and some non-European) laboratories with expertise in the area of physical and biological retrospective dosimetry. The aims of WG10 include: establishing a multi-parameter approach to dose assessment in retrospective dosimetry (including emergency response); evaluating newly developed dosimetry methods; and establishing a common approach for uncertainty estimation throughout biological and physical methods of dosimetry. In order to address this last point, EURADOS WG10 task group 10.6 was created, with a focus on uncertainties associated with retrospective dosimetry techniques.

Uncertainty analysis can be defined as the method to quantify the degree of confidence considering the model outputs taking into account the model inputs (data and parameters) ${ }^{18}$. In classical statistics, the aim of uncertainty assessment is to provide a range around the estimated quantity, the uncertainty interval, within which the real value of the variable is likely. The uncertainty interval usuallydepends on the standard deviation that corresponds to the uncertainty associated with the estimate of the variable. The process of uncertainty assessment is an intrinsic part of any method of retrospective radiation dosimetry, however, it has previously been noted that estimation approaches vary between different dosimetry techniques and, furthermore, that the overall effort devoted to uncertainty analysis varies widely between groups of retrospective dosimetry practitioners ${ }^{19,20}$. The Guide to the Expression of Uncertainty in Measurement (GUM) is the standard reference for uncertainty analysis techniques, and most of the retrospective dosimetry laboratories across Europe and the world use the GUM 


\section{RETROSPECTIVE DOSIMERTY UNCERTAINTIES}

uncertainty estimation techniques ${ }^{21}$. There are several relevant ISO standards and guides to assist with implementation of $\mathrm{GUM}^{22,23}$ and the associated analysis techniques, e.g. for assessment of accuracy ${ }^{24}$ and laboratory intercomparisons ${ }^{25}$.

There are also a number of alternative techniques that may be employed, both for measurement and calculation of the quantity of interest, i.e. absorbed dose, for the propagation of uncertainties through this calculation, and for quantification of both type A (generally corresponding to random) and type B (generally systematic) uncertainty estimates. For example, Monte Carlo simulation, which includes autoand cross-correlations among the components, can be a convenient means of obtaining probability distributions for parameter estimation in non-linear systems ${ }^{26,27}$. This can be combined with the use of Bayesian logical constructs to constrain parameter estimation to help improve the accuracy of dose estimates ${ }^{28}$. Bayesian statistics considers the uncertain parameters and variables at random (probabilistic variables). The analysis takes into account assumed prior distributions for each parameter (which must be chosen by the user), together with the experimental data to produce a subsequent probability density function (pdf) that describes the probability of the observations. In all cases, the appropriateness of the approach should be carefully evaluated ${ }^{29}$.

The aims of EURADOS WG10 task 10.6 are thus to survey the different methods used to assess uncertainties in retrospective techniques, to compare and contrast the different approaches, to popularise the potential of Monte Carlo or Bayesian techniques, to identify best practice and finally to attempt to homogenise the approaches where possible and/or desirable. The survey of uncertainty assessment methods used by WG10 participants was carried out in 2012. The survey participants were overwhelmingly supportive of the need for a review of uncertainty analysis techniques, inter- and intra-technique comparisons of methods, and organization of centrally administrated training once best practice has been identified.

In this publication, the authors present a comparison of techniques of uncertainty estimation amongst laboratories using biological and physical retrospective dosimetry methods of dicentric chromosome, micronucleus, PCC, FISH translocation and $\gamma$-H2AX analysis, EPR, OSL and TL. The data were collated from the survey and discussions at EURADOS annual meetings 2012 - 2016 and from results of the RENEB networking project ${ }^{17}$. The similarities and differences in recommended uncertainty analysis methods, those used in practise, and also the experimental and external factors that influence the results, are discussed. In biological dosimetry, Bayesian analysis methods have so far only been implemented for the $\mathrm{DCA}^{30}$ and thus are discussed in this context. Bayesian approaches have been developed for application in luminescence retrospective dosimetry per $s e^{28}$, and others are widely used to compare retrospective archaeological and geological age estimates ${ }^{31}$, but have not yet been applied for accident reconstruction. The wider applications of Monte Carlo sampling are also discussed, with the aim of promoting the techniques for use within the community. Finally, areas in which biological and physical retrospective dosimetry uncertainty analysis methods may be improved are considered.

\section{DOSIMETRY METHODS}

There are a number of relevant publications including recent reviews of biological and physical retrospective dosimetry methods, thus we here only present a relatively high level summary in order to set the scene for the review of the uncertainty estimation methods that are currently in use.

\section{Biodosimetry}

The most well-established assays for biological dosimetry rely on assessing chromosome aberrations in peripheral blood lymphocytes. The rationale behind counting aberrations is that the number of aberrations induced by ionising radiation corresponds to the absorbed dose. The established techniques are: (1) the dicentric chromosome assay (DCA, which is the "gold standard"2; (2) Fluorescence in situ hybridization $(\mathrm{FISH})^{4}$; (3) Premature chromosome condensation $(\mathrm{PCC})^{5}$; (4) cytokinesis-block micronucleus assay $(\mathrm{CBMN})^{3}$, and (5) counting of $\gamma-\mathrm{H} 2 \mathrm{AX}$ foci which form at the site of double strand breaks ${ }^{6}$.

The two main prerequisites for biodosimetry are the stability of aberrations with time following irradiation and knowledge of the background level, i.e. the number of aberrations in a given sample before irradiation occurred. DCA, PCC and CBMN show stability of responses for several months, as induction of these aberrations means the cells cannot reproduce and thus the cells die and these aberrations are gradually removed from the population of circulating lymphocytes, Therefore, the relative specificity to ionising radiation drives the accuracy of these methods. In contrast, $\gamma-\mathrm{H} 2 \mathrm{AX}$ and $\mathrm{FISH}$ are not radiation specific, however, the individual background levels are becoming more well understood ${ }^{4,6,32}$. The vast majority of $\gamma$-H2AX aberrations disappear within approximately 24 hours and thus this is very much a short term assay, however, translocations detected by FISH are stable over many years. All of the established methods were developed based on visual scoring techniques, and a 


\section{AINSBURY ET AL}

large amount of work has been required to ensure their suitability for mass casualty events ${ }^{17}$. The current status is promising although the need for automation and other more rapid strategies remains ${ }^{1,33,34}$ and there is also a need for development of appropriate, reliable, uncertainty methods.

\section{Dicentric chromosome assay}

As the "gold standard" of biodosimetry, the statistical analysis methods for the dicentric chromosome assay (DCA) are extremely well defined. An ISO standard has been created for the assay in routine ${ }^{35}$ and rapid assessment "triage" ${ }^{36}$ modes. The assay's standardised guidance text, the International Atomic Energy Authority (IAEA) cytogenetics manual ${ }^{1}$, is used by almost all practitioners of the assay for biodosimetry purposes.

In brief, the dicentric yield $y$ (average number of dicentric chromosomes per cell) is modelled as a function of the absorbed dose $D$ by formation of a linear or linear-quadratic calibration curve:

$$
y=C+\alpha D+\beta D^{2}
$$

The specific type (energy/LET) of radiation determines the type of curve, which is created by counting (scoring) aberrations in large numbers of cells, especially at low doses. For in vivo exposure cases, the yield of aberrations observed in the exposed sample is then compared to the calibration curve. Dicentrics are highly specific to ionising radiation, with only a few rarely used radiomimetic drugs able to induce them, thus, the lower limit of detection is approximately 100 $\mathrm{mGy}^{1}$. Simple methods also exist to account for fractionation or partial-body exposures, chiefly based on adherence to or departure from the expected Poisson distribution of aberrations ${ }^{1}$.

\section{Micronucleus assay}

The in vitro cytokinesis-block micronucleus assay $(\mathrm{CBMN})$ is another well-established method for biodosimetry ${ }^{3}$. The assay is based on assessing the frequency of acentric chromosome fragments (micronuclei, $\mathrm{MN}$ ) and a small extent malsegregation of whole chromosomes in binucleated (BN) cells. MN are also caused by many clastogenic and aneugenic agents ${ }^{37}$ and thus are not specific for ionising radiation. Compared to the DCA, scoring of $\mathrm{MN}$ is simple and quick. Furthermore, scoring can be automated and international standardization is in place ${ }^{38}$.

The reported background frequency of $\mathrm{MN}$ is variable: values ranging from 0 to 40 per $1000 \mathrm{BN}$ have been recorded ${ }^{1}$. Consequently, the lower limit for dose detection by conventional CBMN is $0.2-0.3 \mathrm{~Gy}$; although more detailed analysis restricted to centromere positive cells lowers this limit to $0.05-0.1$ Gy. The most important influencing factors of MN background are dietary factors, exposure to environmental clastogens and aneugens, age and gender ${ }^{1}$. When possible, for instance for medical exposure scenarios, the variability of the background level should be decoupled from the other parameters by identification of the individual background level in blood samples taken before irradiation ${ }^{39}$.

Dose estimation using CBMN follows the same strategy as for the DCA. The absorbed dose can be assessed up to few months after exposure ${ }^{1,40,41}$. A drawback of CBMN is the natural overdispersion of $\mathrm{MN}$ data, therefore partial-body irradiation is hard to detect, and also the larger variation in background levels which means that the detection limit is generally greater $^{37}$.

\section{$P C C$}

An important limitation of the DCA, CBMN and FISH assays is the prerequisite for lymphocytes to enter the mitotic phase, which requires culturing for 48 hours or more. Thus dose estimates always take several days. The need for metaphases induces several technical problems including radiation induced mitotic delay and cell death that can lead to non-representative cell samples. After high doses of ionising radiation, this can cause considerable underestimation of absorbed doses. Premature Chromosome Condensation (PCC) can be induced by cell fusion or chemical induction. The cell fusion PCC technique visualises chromosome aberrations in the interphase cells, which can allow same day biological dose assessment. Chemically induced PCC has been validated for triage following in several potential exposure scenarios ${ }^{1,5,40}$.

PCC analysis is not a biomarker on its own, rather it should be combined with scoring of specific chromosome aberrations (e.g. fragments, rings or translocations). The frequency of spontaneous occurring PCC fragments is in the range of the dicentric frequency, 1 to 3 in 1000 cells.

For dose assessment with PCC, the same tools are used as presented for the DCA. PCC assay is particularly useful for assessment of a wide range of doses. It is applicable as well to exposure at low doses (as low as 0.2 Gy for PCC fragments) as to lifethreatening high acute doses of low and high-LET radiations (up to $20 \mathrm{~Gy})^{42}$.

\section{Fluorescence in situ hybridization}

Dicentric chromosomes, rings and MN are "unstable" chromosome aberrations and thus they vanish from peripheral blood lymphocytes pool at the rate that cell 
renewal occurs. Fluorescence in situ hybridization (FISH) techniques allow identification of stable translocations, and have been used for many years for assessment of past exposures ${ }^{1,43}$.

However, background frequencies increase significantly with age and vary greatly between individuals of the same age and dose history. No significant gender effects have been observed but smoking habit has been suggested to be of significance $^{32}$.

The observed number of aberrations must be corrected for these known confounding factors in order to obtain radiation-induced translocations only, before dose response curves are calculated as for dicentrics ${ }^{1}$. Note that as a consequence of stability and nonspecificity to radiation, the minimum detectable dose is limited as a function of time: in the region of $1.8 \mathrm{mGy}$ per year (20 - 69 years) for acute doses, and for chronic exposure $15.9 \mathrm{mGy}$ per year respectively ${ }^{42}$. At high doses, correlation of translocations and complex aberrations in cells is also of importance ${ }^{1}$. Although the biological complexity of this assay is relatively well understood, the uncertainty analysis techniques remain simplistic and more work is needed.

\section{$\gamma-H 2 A X$}

The $\gamma-\mathrm{H} 2 \mathrm{AX}$ assay, commonly used for investigating radiosensitivity, has in recent years become a wellestablished biomarker for radiation induced DNA double-strand breaks and thus radiation exposure ${ }^{6,44}$. Fluorescence microscopy or flow cytometry measure the formation of DNA repair-protein clusters called $\gamma$ $\mathrm{H} 2 \mathrm{AX}$ foci (in terms of number or intensity respectively) in peripheral blood lymphocytes of exposed persons ${ }^{45,46}$. The foci are not specific for radiation exposure and spontaneous frequency is very low. Wide use for biodosimetry purposes is limited by fast loss of the signal (maximal $\gamma-\mathrm{H} 2 \mathrm{AX}$ level is reached $30 \mathrm{~min}$ after irradiation, tissue related the halflife of the signal is $2 \mathrm{~h}-7 \mathrm{~h}$ ) as well as high individual variability $42,47,48$

Advantages of the assay are its high sensitivity and relatively low detection limit (as low as tens of $\mathrm{mGy}$ ), the need of only a few drops of blood, absence of required lymphocyte cultures and the ease of automation. However, the absorbed dose can only be assessed up to approximately 1 day after exposure ${ }^{49}$. In addition, the influence of factors such as age, gender and genotypes are not yet well understood ${ }^{50-56}$. The relatively large uncertainties allow $\gamma-\mathrm{H} 2 \mathrm{AX}$ to be used for biodosimetry only in extremely controlled scenarios ${ }^{57}$ and uncertainty assessment is carried out on a case-by-case basis, with the sophistication of the analysis varying greatly between laboratories.

\section{Physical retrospective dosimetry}

For the purposes of retrospective dosimetry, physical dosimetry methods are retrospective dose estimation techniques based on the quantitative evaluation of detectable changes induced by ionising radiation in inert materials or by the activation of atoms such as sodium or phosphorus when exposed to neutrons. They are usually only suitable for detection of external exposure and for situations of partial or localised exposure, they provide a useful dosimetric information only if the "fortuitous dosemeter" (defined as a material validated for dosimetry which the individual happens to be carrying) is by chance within the radiation field.

The three physical dosimetry techniques considered in this review are the Electron Paramagnetic Resonance (EPR), the Optically Stimulated Luminescence (OSL) and the Thermoluminescence (TL).

\section{Electron paramagnetic resonance}

EPR dosimetry is based on the quantification of paramagnetic species (defects or free radicals) induced by ionising radiation. In solids, as crystalline materials, the radicals/defects can be trapped and are thus generally sufficiently stable to be measured. The EPR signal is a measure of the radical/defect concentration within the solid matrix and is usually proportional to the mean absorbed dose in the sample. The principle of EPR spectroscopy may be found in several textbooks ${ }^{58-}$ 60 and a detailed description of applications in retrospective dosimetry is given by Trompier and colleagues $^{61}$. In general, depending on the complexity of the EPR spectra, the area under the absorption curve or peak-to-peak amplitude of an EPR signal are used and considered to be proportional to the concentration of paramagnetic species.

The validated assays for retrospective analysis are calcified tissues (tooth enamel and bone) and sugar, while materials such as nails, mineral glass, sweeteners, plastics and clothing fabrics that are widespread among humans are under investigation $^{12,13,62-66}$. The ideal characteristics of a beneficial fortuitous dosemeter are a high radiationinduced signal yield, the absence of endogenous signals, a low UV-induced signal, low detection limit, the linearity of the signal with dose and post-irradiation signal stability ${ }^{61}$. The quantity of the radiation-induced radicals may be correlated to a value of the absorbed dose either via application of a "positive control" - the delivery of additional radiation doses with a laboratory source ("additive dose" method) or via a calibration curve. Calibration curves may be created on an individual basis for each sample, or using different samples each irradiated at a different radiation dose, 


\section{AINSBURY ET AL}

i.e. always applying the same sample-specific, signalto-dose calibration curve. A disadvantage of the universal calibration curve is that it does not take into account the specific sensitivity of the sample. The curve is built using the average of the sensitivities of different samples. This of course affects the uncertainty associated with the estimated dose.

When EPR is used for ionising radiation dosimetry, other confounding factors such as UV exposure or mechanical stress may generate additional radicals whose signals may overlap or mask the radiationinduced signal. In these cases, spectral simulations or other numerical analysis methods are needed, in order to decompose the different components of the spectrum and extract the signal of interest.

The average lifetimes for the radicals vary from minutes to billions of years. Controlling the sample storage condition, for example by keeping the samples in dark, low humidity and sometimes in a freezer, will save the samples from unwanted changes.

A key advantage of EPR analysis is that it can be repeated as many times as needed, as the readout process does not alter the signal. This gives the possibility to estimate the effect of sample positioning, spectrometer reproducibility and stability which all play a large role in the uncertainty budget for EPR dose estimates.

\section{Optically/thermally stimulated luminescence}

Luminescence dosimetry relies on the stimulated emission of light from an insulator or a semiconductor after the absorption of energy from ionising radiation ${ }^{67}$. Ionising radiation transfers energy to the electrons of the solid, moving them to a metastable state. When the electrons return to the ground state, recombination occurs and luminescence light is emitted. This recombination occurs after absorption of stimulation energy provided by heat in the case of Thermoluminescence (TL) and by light for Optically Stimulated Luminescence (OSL).

Crystals contain defects, which produce spatially localised energy levels in the energetically "forbidden" zone between the valance and conduction bands. Ionising radiation produces electron-hole pairs by exciting electrons beyond the potential of their parent molecule into a delocalised state, which is most commonly the conduction band. As electrons and holes migrate in the conduction and valence bands, most recombine rapidly but some become trapped in metastable states associated with the defects. Later, these can be excited by thermal or optical stimulation, so that electrons and holes are again able to recombine. Following recombination, the host molecule is excited, and some emit photons at visible wavelengths as they de-excite: this emission is termed thermally or optically stimulated luminescence, depending on the type of stimulation.

In TL, the light yield is recorded as a glow curve, i.e. as a function of the stimulating temperature, whereas in OSL the number of emitted photons per time interval is recorded as a function of the optical stimulation time. For both stimulation modes, the area under the glow curve / OSL decay curve is related to the total number of emitted photons and thus to the absorbed dose in the dosemeter.

Published results on the applications of TL and OSL for retrospective dosimetry have concentrated on OSL and TL techniques on chip cards, electronic components and glass from personal electronic devices $^{68}$. For some of these methods, interlaboratory comparisons have been performed ${ }^{69}$.

\section{UNCERTAINTY ESTIMATION APPROACHES}

\section{Biological dosimetry - dicentric assay}

\section{Frequentist approach: Confidence limits}

Uncertainty assessment for cytogenetic dosimetry is widely understood as the quantification of the variability within the dosimetric model, e.g. as defined by equation (1). Thus, parameter uncertainties as well as biomarker variability need to be considered. Indeed, full uncertainty analysis for cytogenetic dosimetry following the $\mathrm{GUM}^{21}$ considers a long list of factors. For routine dosimetry, this list includes: the type and parameters of the dose response curve, the stochastic characteristics of the biological marker, inter- and intra-individual variability, technical noise sources and practical limitations (e.g. in vitro calibrated methods applied to in vivo data). More complex scenarios of exposure induce further challenges, as described by Vinnikov and colleagues ${ }^{19,70}$. As processing is time consuming and the level of experience varies between laboratories, the potential for standardization and verification of uncertainty analysis methods is very limited. This may explain the absence of agreement on some of the expected uncertainty parameters within this field, such as the coverage factor ${ }^{21}$.

For the DCA, which has the most well developed set of uncertainty estimation methods of all the biological assays, uncertainties of estimated doses are generally assessed by the analysis of the variability of the dicentric yield $y$ and the parameters of the calibration curve $C, \alpha$, and $\beta^{71-74}$, according to the GUM methodology ${ }^{21}$ (with detailed examples for several of the biological techniques given ${ }^{75}$ ). In the version of the IAEA manual published in 2001, which was the first time uncertainty analysis was discussed in detail in a methodological biodosimetry publication, three 
methods of uncertainty assessment were presented, labelled A - C. It was reasoned that Merkle's Approach "C" $\mathrm{C} 72$ performs best for low numbers of dicentrics (as in low doses and/or few cells), whereas for high doses Savage's Approach " $A$ " 71 is more precise. In the updated 2011 version of the IAEA manual ${ }^{1}$, Merkle's approach is discussed in greater detail. This method of uncertainty assessment allows incorporation of both the Poisson error of the yield as well as errors in the dose response curve parameters. The confidence bands of the calibration curve follow from the insight that the maximum likelihood estimate of the parameter vector is asymptotically multivariate normal ${ }^{72}$. The upper limit $y_{u l}$ and lower limit $y_{l l}$ for the expected mean yield are therefore:

$$
\begin{aligned}
& y_{u l / l l}=C+\alpha D+\beta D^{2} \\
& \pm R \sqrt{ }\left(s_{C}{ }^{2}+s_{\alpha}{ }^{2} D^{2}+s_{\beta}{ }^{2} D^{4}+2 s_{C, \alpha} D+2 s_{C, \beta} D^{2}+2 s_{\alpha, \beta} D^{3}\right)
\end{aligned}
$$

where $s_{x}^{2}$ denotes the variance of $x$ and $s_{x, z}$ the covariance of $x$ and $z$, for each value of $x$ and $z$ in the equation. The regression factor $R^{2}$ gives the range within which the true average yield is to be expected. This is equivalent to the confidence limit of the Chisquared distribution with 2 or 3 degrees of freedom for linear or linear-quadratic fits; i.e. for $95 \%$ confidence intervals, $R=2.45$ at 2 degrees of freedom $(d f)$ and $R=$ 2.80 at $d f=3$, respectively.

Finally, the calculation of confidence intervals for the estimated dose includes two steps:

(1) Determination of the boundaries $Y_{U}$ and $Y_{L}$ of the dicentric yields which are consistent with the observed yield in the sample $(95 \%$ confidence limits for the mean parameter of a Poisson random number).

(2) Determination of the absorbed dose for which the upper dose response curve $y_{u l}$ exceeds $Y_{L}$ and the dose where the lower curve $y_{l l}$ exceeds $Y_{U}$.

Note that some authors reason that the combination of 95\% confidence intervals for the dicentric yield as well as for the confidence bands leads to a falsely large confidence interval for the dose, thus in case of combined errors $83 \%$ confidence limits of the Chisquared distribution are more appropriate ${ }^{76,77}$ (the square root of the regression factor at $1-\alpha=83 \%$ is $R=$ $1.88(d f=2)$ and $R=2.24(d f=3)$ respectively).

The alternative approaches given in the IAEA manual: A (Savage ${ }^{71}$ ) and B (again Merkle ${ }^{72}$ ), are built on classical error propagation calculations for normally distributed random variables. They apply the Delta method to calculate the standard error for the estimated dose from the calibration curve and its parameter uncertainties.

For dose estimations in more complicated scenarios, extensions exist including correction for protracted or fractioned doses and partial-body exposures. In these cases, in principal, after correction of the curve, uncertainty assessment follows the same strategy as above. However, in this case, either the simplified method $\mathrm{C}$ described above is used (with no parameter uncertainties) or equation (2) must be adjusted manually. Tools to apply the standard (IAEA) methods for automated dose estimation and uncertainty assessment are available, including CABAS (Chromosomal ABerration Calculation Software) ${ }^{78}$ and DoseEstimate $^{79}$.

In order to assess inhomogeneous exposures more realistically, Sasaki modelled the damaged cell population with a mixed Poisson, which can be numerically deconvoluted ${ }^{80}$. The resulting exposure profile indicates some uncertainty within the dose. However, this does not represent a rigorous uncertainty assessment.

In addition, a correction factor for confidence intervals of overdispersed data (i.e. dispersion index $\left.\sigma^{2} / y>1\right)$ is proposed ${ }^{1}$. For those samples, the limits of the expected range of the yield, $Y_{U}$ and $Y_{L}$, of a sample with mean $y$ and variance $\sigma^{2}$ should be adjusted as follows (with either $Y_{U}$ and $Y_{L}$ as appropriate):

$$
Y_{U / L}^{*}=Y_{U / L}\left(\frac{Y_{U / L}}{y}\right)^{\sqrt{\frac{\sigma 2}{y}}}
$$

\section{Probabilistic Approach: Bayesian methods}

In parallel to the classical, frequentist approach, Bayesian methods are becoming increasingly popular $^{30,81,82}$. Key to the Bayesian concept is the application of the inversion theorem in its continious version, i.e.:

$P\left(D \mid X_{o b s}\right)=\frac{P\left(X_{o b s} \mid D\right) P(D)}{\int P\left(X_{o b s} \mid D\right) P(D) d D} ;$

where $D$ denotes the unknown parameter (absorbed dose) and $X_{o b s}$ the observation (the dicentric yield within the sample, $y$, and the calibration data). Thus, the posterior dose distribution (or calibrative dose density), $P\left(D \mid X_{o b s}\right)$, scales with the product of the likelihood (or predictive density) $P\left(X_{o b s} \mid D\right)$ and the prior $P(D)$ :

$$
P\left(D \mid X_{o b s}\right) \propto P\left(X_{o b s} \mid D\right) P(D)
$$

With respect to uncertainty analysis, the Bayesian approach does not require additional considerations, since the resulting distribution $P\left(D \mid X_{o b s}\right)$ (probability for a dose given the data) inherently provides quantification of the uncertainty within the dosimetric 


\section{AINSBURY ET AL}

model. Consequently, Bayesian uncertainty intervals for the calibration parameter (the dose in this case) are accurate.

Apart from the intrinsic inclusion of uncertainty within the posterior model, the greatest advantage compared to the frequentist approach is the possibility to include other information besides the number of aberrations through the chosen prior distribution(s). The choice of the prior could be sensitive, since well chosen, informative priors should guide noisy data towards the true dose, whereas incorrect priors may drive the estimate away from the true dose. Some authors reason that high quality prior information is almost always available, and thus Bayesian approaches are more appropriate for biological dosimetry ${ }^{70,81}$. Higueras and colleagues ${ }^{30}$ also showed that if an appropriate prior is sensely determined, the actual choice of prior in fact doesn't greatly impact the overall dose assessment in some scenarios.

In contrast to the frequentist approach, which relies on processing the information successively from the initial sample, to the calibration data, to a point estimate of the dose surrounded by a confidence interval; the Bayesian approach incorporates all decisions at the same time and the resulting equation is then analytically, numerically or empirically solved for the required components. A fully Bayesian method thereby also adjusts the probability density function of the aberrations to the specific scenario of exposure and simultaneously incorporates the uncertainties of the parameters of the dose response curve. The mathematical complexity of this task means that it is not possible to define a general Bayesian solution applicable to all exposure scenarios.

Nevertheless, Di Giorgio and Zaretzky showed a procedure to include prior information in dose estimation in a Bayes-like manner ${ }^{83}$. Discretization of the dose range and a separated frequentist estimation of response curve parameters provides a straightforward method resulting in a Bayes-like posterior of the $\operatorname{dose}^{83}$. Note that this example also illustrates the influence of the prior on the credible intervals for the dose.

For Bayesian uncertainty assessment of the dose, three types of solutions can be identified from within the literature. Firstly, analytical expressions for simplified scenarios: The earliest prominent example of such a solution is the calibrative density for a Poisson distributed number of aberrations linked to the absorbed dose via a linear dose response without intercept ( $C=\beta=0$ ) using Gamma priors for dose and slope $^{84}$. In this case, an analytical expression is derived that is proportional to the posterior. The authors reasoned that the trivial dose response curve is appropriate for neutrons (high LET) at high doses, however, neutrons are known to imply overdispersion $^{85}$. Brame and Groer revisited the same scenario in 2002, replacing the Poisson distribution by a negative binomial in order to jointly model the density of the slope and the degree of overdispersion ${ }^{82}$.

Secondly, practical guides for specific scenarios that provide $\mathrm{R}$ code for reuse. Higueras et al. ${ }^{39}$ discussed the reasonable set up of Poisson and compound Poisson models (Neyman A, negative binomial, Hermite) for biodosimetry. Complete and simplified models are provided and three examples are given for dose assessments for a linear quadratic calibration curve (two Poisson and one negative binomial regression model). For each example three different priors are compared $^{39}$. Together with Vinnikov, the same group of authors presented a guide for analysis of partialbody exposure for a zero-inflated Poisson model ${ }^{30}$. This guide approximates credible intervals for the irradiated fraction of the body and the received dose simultaneously from:

$$
\begin{aligned}
& P\left(D, F, d_{0} \mid y\right) \\
& \propto\left(F e^{-\frac{D}{d_{0}}}-F\right. \\
& +1)^{-n} \sum_{j=1}^{n_{0}}\left(\begin{array}{c}
n_{0} \\
j
\end{array}\right) \frac{F^{n-j}(1-F)^{j}}{(n-j)^{s}} P\left(X_{j}=s \mid D\right) P(D) P(F) P\left(d_{0}\right)
\end{aligned}
$$

where $D$ is the absorbed dose, $F$ the fraction of irradiated body, $d_{0}$ the $37 \%$ cell survival dose, $n$ number of cells in patient data, $\mathrm{n}_{0}$ the number of cells without aberrations, and $X_{i}$ is a negative binomial distributed random number corresponding to the unirradiated fraction of the cells whose mean and variance depends on the index $i$ and the mean and the variance of the calibration curve respectively. Uniform priors for $F$ and $d_{0}$ and a Gamma prior for the dose are used $^{30}$

Thirdly, Software packages: The Java application CytoBayesJ for cytogenetic radiation dosimetry ${ }^{81}$ and $\mathrm{R}$ package radir containing the models by Higueras ${ }^{86}$ offer platform independent software solutions to Bayesian uncertainty assessment. CytoBayes offers tools for (i) distribution testing (compound Poisson models), (ii) posterior calculations of the number of aberrations (several combinations of priors and yield models), (iii) Bayesian-like dose assessment (Poisson data), (iv) full Bayesian calculation of posteriors of the dose (Poisson data in $y=\alpha D$ ), as well as (v) Bayesian methods for detection limits ${ }^{81}$. In order to simplify the analysis, most scenarios include a Bayesian uncertainty assessment of the dicentric yield and then make a conventional frequentist inverse regression step due to mathematical complexity. 
Ultimately, it can be concluded that the Bayesian methodology provides the most coherent approach, but at the same time it is far more technically challenging than the dose and uncertainty assessment methods currently recommended and used by most practitioners ${ }^{1}$. Despite recent developments, such methods thus remain to date an "expert" tool. Therefore, software solutions such as those described above will be required to bridge the gap between the necessary mathematical skills and the users. In particular, the potential pitfall of incorrectly chosen priors will need careful consideration going forward, since the methodological coherence of prior and posterior can be seen conceptually as a self-fulfilling prophecy that masks unexpected results.

\section{EPR}

\section{Sources of uncertainty}

Uncertainties or "errors" can be classified into two types: type A errors can be evaluated by statistical methods whereas type B errors are commonly termed "systematic errors" and must be dealt with by other means. Historically uncertainties have also been classified as "random" or "systematic" errors, and these terms are still sometimes used for type A and B errors, respectively. However, it is important to note that the GUM recommends the nomenclature of "type A" or "type B" to classify how an error is dealt with rather than where it originates ${ }^{21}$.

For EPR, determination of irradiation doses retrospectively is not a straightforward task. Uncertainties both of type A and of type B are introduced and must be carefully analysed and reported. Several technical publications have been produced, dedicated to the determination of uncertainties with EPR spectroscopy on materials such as tooth enamel or alanine ${ }^{87-90}$. A list of possible sources of uncertainties has been drafted for tooth enamel dosimetry in Fattibene and Callens ${ }^{91}$ and many issues considered in the list (effect of sample anisotropy, parameters of spectrum acquisition, spectrometer instability, sample mass, spectrum processing methods, uncertainty linked to the dose calibration curve, etc.) are valid for almost all the EPR dosimetry methods. As recommended by IAEA ${ }^{87}$, the total combined uncertainty is expressed as the quadratic sum of the possible source of uncertainties, under the assumption that these sources are uncorrelated. Specifically,:

$$
\sigma_{E D}=\sqrt{\left(\sigma_{F}^{2}+\sigma_{S}^{2}+\sigma_{E}^{2}+\sigma_{C}^{2}+\sigma_{T}^{2}\right)}
$$

where $\sigma_{F}$ is the contribution from the fading correction; $\sigma_{S}$ is the contribution from the sample preparation; $\sigma_{E}$ the contribution from the EPR measurement; $\sigma_{T}$ the contribution from the numerical treatment of spectra, and $\sigma_{C}$ is the contribution from the calibration of EPR dose response, including differences in radiation sensitivity.

The fading contribution depends on the detector materials used in analysis. For tooth enamel it can be assumed that $\sigma_{F}$ doesn't contribute to the overall uncertainty ${ }^{87}$, however, a sufficient delay should be observed after irradiation for signal stabilization due to recombination of short-lived species. A minimum delay of 48 hours is usually recommended for calcified tissue. This recommendation is also valid for most irradiated materials including sugars, for example, for which the stabilization delay can reach weeks ${ }^{92}$.

EPR dosimetry is usually not performed on unstable species. Nevertheless, a few groups are considering the use of the unstable signal component in nails for dosimetry application ${ }^{10,11,93}$. In this case, the fading correction may significantly contribute to the uncertainty budget, because of the influence of multiple parameters (temperature humidity, light, etc.) which may be difficult to evaluate for the delay between irradiation and sample harvest. In addition, special attention must be paid to control fading during the storage period or at least the parameters of influence. Similarly, the sample preparation approach depends on the material used as well as on the method chosen.

The other three factors affecting the total uncertainty stem from measurements and data processing. The EPR measurement uncertainty, $\sigma_{E}$, depends on a complex combination of uncertainties linked to the performance of EPR spectrometers per se and the experimental setup. Uncertainty contributions from the numerical treatment of spectra, $\sigma_{T}$, and contributions from the calibration of EPR dose response, $\sigma_{C}$, can be minimised through experimental validation of the method for different materials as has been done for tooth enamel ${ }^{91}$ and smart phone touch screen glass ${ }^{13}$. For this reason, international interlaboratory comparisons of EPR dose reconstruction are the most useful tools for identifying contributing sources of uncertainties and finding the best solution to minimise these.

\section{Evaluation of uncertainties}

The uncertainty analysis approach used by several EURADOS partners is the standard one recommended for uncertainty estimation for EPR on alanine. In brief, this method consists of taking the mean value of multiple measurements as the best evaluation of the true value and the sample standard deviation as the uncertainty on the signal. A minimum of ten measurements is commonly used; however, some EURADOS WG10 members who completed the survey regularly perform 12 or 16 measurements (3 


\section{AINSBURY ET AL}

dosemeters in 4 different orientations or 4 dosemeters in 4 orientations). In order to measure unknown absorbed doses, a calibration curve is required to be created to describe the relationship between EPR signal $(y)$ and dose $(D): y=F(D)$. The parameters of the function, $F$, are estimated through a best-fit procedure. The dose-signal relation is usually linear and weighted regression is sometimes, but not always, applied. Thus, the general expression of the estimated dose ED as a function of the measured signal, $y$, is as follows:

$$
\begin{aligned}
& E D=\frac{y-b}{a} \pm u(E D)= \\
& =\frac{y-b}{a} \pm \sqrt{\frac{1}{a^{2}} u^{2}(y)+\frac{1}{a^{2}} u^{2}(b)+\frac{(y-b)^{2}}{a^{4}} u^{2}(a)+2 \frac{(y-b)}{a^{3}} u(b, a)+u_{c a l}^{2}}
\end{aligned}
$$

where $a$ is the calibration curve slope and $b$ is the yaxis offset. The combined variance $u^{2}(\mathrm{E} D)$ consists of the variance in the measured signals $u^{2}(y)$, the variance in the y-axis offset $u^{2}(b)$, the variance in the slope in the calibration curve $u^{2}(a)$, the covariance of $b$ and $a$ and finally the dose dependent variance in the doses given to the calibration samples, $\mathrm{u}_{\text {cal }}^{2}$. The covariance term was found to be negligible in accurate EPR dosimetry $^{94,95}$ and it is assumed that this will be the case for retrospective dosimetry.

An unbiased estimate of the variance of the measured signals can be obtained from:

$$
u^{2}(y)=\frac{1}{n-2} \sum_{k=1}^{n}\left(y_{k}-b_{k}-a \cdot D_{k}\right)^{2}
$$

where $D_{k}$ are the known absorbed doses given to the calibration samples, $y_{k}$ are the corresponding signal values and $b_{k}$ is in this case the zero dose for each calibration sample. The denominator represents the number of calibration points minus the two degrees of freedom.

Standard regression analysis yields:

$$
\begin{aligned}
& u^{2}(b)=\frac{u^{2}(y) \sum_{k=1}^{n} D_{k}^{2}}{n \cdot \sigma^{2}(D)} \\
& u^{2}(a)=\frac{u^{2}(y)}{\sigma^{2}(D)}
\end{aligned}
$$

where:

$$
\sigma^{2}(D)=\sum_{k=1}^{n}\left(D_{k}-\bar{D}\right)^{2}
$$

and the covariance term is omitted.
These principals allow deduction of chiefly type A uncertainties. Type B uncertainties are generally considered by taking into account uncertainties in fading, corrections for radiation energy, environmental factors, spectrometer variations and calibration dose. Note, however, that fading could be treated as a type A uncertainty if multiple measurements of dispersion in fading for a given time period - such a method is used for TL/OSL dosimetry, as described in the next section.

An alternative approach relies on determination of the absorbed dose in alanine measurements from the calibration curve, by relating the amplitude of the EPR signal to absorbed dose. To estimate the uncertainty of dose, $u(E D)$, an imperfect calibration curve is designed and the procedure described by Nagy ${ }^{89}$ is applied; in the case of a calibration plot based on $n$ calibration points, the confidence interval for the dose value $D$, determined from $m$ replicate measurements of the signal of a test sample, is calculated using the following expression:

$$
D=D_{0} \pm t_{n-2, P} \cdot \frac{s_{f i t}}{b} \cdot \sqrt{\frac{1}{m}+\frac{1}{n}+\frac{\left(D_{0}-D_{\text {mean }}\right)^{2}}{\sum_{i=1}^{n}\left(D_{i}-D_{\text {mean }}\right)^{2}}}
$$

where: $t_{n-2, P}$ is the Student coefficient for the chosen probability $P ; s_{f i t}$ is the standard uncertainty of the mean of the fit; $b$ is the slope of the regression; $D_{0}$ is the dose, $D$, value to be determined and $D_{\text {mean }}$ is the mean of the $D$ values of all calibration point $D_{i}$.

\section{Performance parameters and predicted uncertainty}

In the framework of the European Research project Southern Urals Radiation Risk Research (SOUL, 2005), a benchmark protocol was established between three EPR laboratories (HMGU, Munich, ISS, Rome, and IMP, Ekaterinburg) for the definition of the performance parameters for EPR dosimetry with tooth enamel and for the prediction of associated uncertainty $^{96}$. The parameters critical dose and limit of detection, taken from chemical metrology ${ }^{97,98}$ were deemed to be most appropriate to characterise the uncertainty of EPR measurements.

The definition of critical dose follows from the hypothesis test for $95 \%$ probability of an unirradiated sample and hence allows a false positive error rate, $\alpha$, of $5 \%$. In other words, within the distribution of measured EPR signal amplitudes from unexposed samples, there is an accepted probability of 5\% that the amplitude is larger than the critical amplitude, which is the decision limit below which it is assumed that the sample was not exposed and above which it is assumed that an exposure occurred. The absorbed dose value 


\section{RETROSPECTIVE DOSIMERTY UNCERTAINTIES}

corresponding to the critical amplitude on the EPR signal-to-dose response curve is then termed the critical dose.

The definition of the limit of detection follows from the hypothesis test for $95 \%$ probability that the sample was exposed, hence allowing for a false negative error rate $\beta$ of $5 \%$ indicating that an exposure did not occur. That is, within the distribution of the measured EPR signal amplitudes from exposed samples, there is probability $\beta$ of $5 \%$ that the amplitude is lower than the critical amplitude. A graphical illustration of the definitions of critical amplitude and limit of detection is shown in Figure 1.

$<$ Figure 1 here $>$

Figure 1. A graphical illustration of the definitions of critical amplitude $\left(I_{C L}\right)$ and critical dose $\left(D_{C L}\right)$, limit of detection of signal amplitude $\left(I_{D L}\right)$ and of absorbed dose $\left(D_{D L}\right)$. (Figure adapted from ${ }^{96}$ ).

The critical amplitude, $I_{C L}$, and the limit of detection, $I_{D L}$, of EPR signal intensity are calculated from the mean of measurements of unexposed samples $\left(b_{0}\right)$ and the estimated standard deviation of $n$ EPR measurements of unexposed samples, $\hat{\sigma}_{0}$, and samples exposed to a dose $D_{D L}$. $\hat{\sigma}_{D L}$, respectively:

$$
\begin{aligned}
& I_{C L}=b_{0}+t_{(1-\alpha, n-2)} \hat{\sigma}_{0} \\
& I_{D L}=I_{C L}+t_{(1-\beta, n-2)} \hat{\sigma}_{D L}
\end{aligned}
$$

The estimated standard deviation must be multiplied by the Student's critical value $t_{(1-[\alpha \text { or } \beta], n-2)}$, the (1- $[\alpha$ or $\beta]$ ) percentage point of Student's $t$ distribution with the single-sided confidence interval chosen according to the desired confidence level (1-[ $\alpha$ or $\beta])$ and number of samples $n$. The standard deviations may be evaluated from the $90 \%$ prediction bands of an unweighted linear least-squares fit of the EPR signal-to-dose response curves in the case of constant uncertainty. Alternatively, in the case of dose dependent uncertainty, the values of the standard deviations may be predicted from an analytical model function formulated from the variance of EPR measurements on the absorbed dose. An example function of variance as a function of absorbed dose in tooth enamel, developed at the EPR laboratory of the ISS, is reported in Figure 2.

Following work carried out under the European project SOUL, the benchmark protocol has been used for the estimation of the performance parameters within several EPR dosimetry method intercomparisons $^{13,99}$.
$<$ Figure 2 here $>$

Figure 2. Model function of variance as a function of the EPR signal amplitude in tooth enamel, built at the EPR laboratory of the ISS.

\section{OSL/TL}

\section{Evaluation of uncertainties}

In OSL and TL, there is no specific standard dedicated to the evaluation of uncertainties, and evaluations normally follow the classical $\mathrm{GUM}^{21}$ guidance.

Uncertainty analysis is performed using the standard theory of error propagation. If only a single dose calibration point is used then the unknown absorbed dose, $D_{X}$, is obtained through a simple comparison between the corresponding luminescent signal (TL or OSL) and the luminescent signal, $I_{c a l}$, obtained after exposing the same dosemeter to a calibration dose $\mathrm{D}_{\text {cal }}$. If fading is an issue then either the signal or the measured dose can be corrected for this effect.

Two cases will be considered here, both of which have been applied in the literature: 1) the fading factor is determined individually for the sample in question using the (known) time $t_{X}$ since irradiation and 2) the fading factor is calculated based on a known fading function, with associated uncertainties.

In the case of 1), a possible approach would be as follows: after measurement of the signal $I_{X}$ related to the unknown dose $D_{X}$ of the incident, with a time delay $t_{X}$ since this incident, the sample is given a calibration dose $D_{c a l}$ and a corresponding signal $I_{c a l}$ is measured after a time $t_{c a l}$. The latter procedure is then repeated with the same dose $D_{c a l}$ but this time waiting for a longer time interval $t_{X}$ (the same time delay as for the accidental exposure), before measuring the corresponding signal $I_{X, D c a l}$. The fading factor is then directly determined by the simple relation:

$$
f=\frac{I_{X, D c a l}}{I_{\text {cal }}}
$$

In this case, only the measurement uncertainties of $I_{X, \text { Dcal }}$ and $I_{X}$ are required for evaluation of the uncertainty in $f$. The unknown absorbed dose $D_{X}$ is then calculated as:

$$
D_{X}=\frac{I_{X}}{f \times I_{\text {Cal }}} \times D_{c a l},
$$

with $I_{X}$ being, as above, the signal measured after the unknown exposure, with a delay time $t_{X}$. It is important to note the difference between $I_{X}$ and $I_{X, D c a l}$. Equation 17 can then be simplified to: 


$$
D_{X}=\frac{I_{X}}{I_{X, D c a l}} \times D_{c a l}
$$

Therefore only the measurement uncertainties in $I_{X}$ and $I_{X, D c a l}$ and the uncertainty in determining $D_{c a l}$ are needed for the calculation of uncertainty in $D_{X}$, which can be carried out using the GUM methodology as explained in the above sections.

This method assumes that the uncertainties in $I_{X}$ and $I_{X, D c a l}$ exhaustively explain the observed deviances in the dose-response and fading curves. However, from experience, it is known that this is probably not always the case and the uncertainty in $D_{X}$ is therefore likely to be underestimated using the above procedure. For instance, an uncertainty in the time $t_{X}$ since the unknown exposure is not considered.

In the case of 2), fading is calculated according to a functional relationship fitted to datasets of other samples. For chip cards and electronic components, where the effect of anomalous fading is suspected, this functional relationship between intensity and time since irradiation is well-known ${ }^{69,100-104}$ :

$$
I(t)=I_{C}\left[1-\kappa \ln \left(\frac{t}{t_{C}}\right)\right]
$$

With $I_{C}$ being the signal intensity that would be observed after an (arbitrarily) chosen time $t_{C}$ after irradiation and $\kappa$ a fitting constant (in the literature, the common logarithm is often used and $\kappa$ replaced by $g / 100$, with $g$ being the percentage decrease per decade). If, for convenience, $t_{C}$ is set to $t_{c a l}$ before fitting of equation (19), then the fading factor can be calculated as:

$$
\mathrm{f}=\frac{I\left(t_{x}\right)}{I\left(t_{c a l}\right)}=\left[1-\kappa \ln \left(\frac{t_{x}}{t_{c a l}}\right)\right]
$$

The difference between equations (20) and (16) is that here the signal intensities are calculated rather than measured. If, again, a single calibration dose is used to convert signal to dose, the unknown dose $D_{X}$ is calculated according to equation (17). If uncertainties are assumed in $\kappa, t_{X}, t_{c a l}, I_{X}, I_{c a l}$ and $D_{c a l}$, then in this simplified case, the uncertainty in $D_{X}$ can be assessed using GUM methodology:

$$
\delta_{\mathrm{D}_{\mathrm{x}}}=\mathrm{D}_{\mathrm{x}} \cdot \sqrt{\left(\frac{\delta_{\mathrm{D}_{\text {cal }}}}{\mathrm{D}_{\text {cal }}}\right)^{2}+\left(\frac{\delta_{\mathrm{I}_{\mathrm{x}}}}{\mathrm{I}_{\mathrm{x}}}\right)^{2}+\left(\frac{\delta_{\mathrm{I}_{\mathrm{cal}}}}{\mathrm{I}_{\mathrm{cal}}}\right)^{2}+\left(\frac{\delta_{\mathrm{f}}}{\mathrm{f}}\right)^{2}}
$$

with

$$
\sigma_{f}^{2}=\left(\ln \left(\frac{t_{X}}{t_{c a l}}\right)\right)^{2} \sigma_{\kappa}^{2}+\left(\frac{\kappa}{t_{X}}\right)^{2} \sigma_{t_{X}}^{2}+\left(\frac{\kappa}{t_{c a l}}\right)^{2} \sigma_{t_{c a l}}^{2}
$$

It should be emphasised that if published fading parameters in equation (19) are used and $t_{C}$ does not equal $t_{c a l}$, then equation (20) should be applied in its more general form, as a ratio of two calculated intensities with associated uncertainties. The calculation of the uncertainty in $D_{X}$ is then more laborious but still straightforward. Another issue is that if equation (19) is fitted to fading data obtained from averaged signals of several samples, which is sometimes done, then the calculated signal uncertainty will always be lower than the standard deviation of the input data, i.e. the uncertainties in the parameter $\kappa$ and $I_{C}$ are unlikely to describe the full variability in observed fading behaviour. One possibility to circumvent this issue is to fit the fading data of each sample individually, rather than to average signals and then to calculate average and standard deviation of the group of obtained parameter values. This approach has e.g. been pursued in the MULTIBIODOSE project ${ }^{49}$.

For a luminescence reader with a built-in calibration source, the time delay $t_{c a l}$ between irradiation with the calibration dose and measurement is usually known very accurately, therefore the uncertainty $\delta_{c a l}$ can be neglected in this case. On the other hand, an increase in the value of $t_{c a l}$ leads to a reduction in the first term in equation (22) and thus to a reduction in the uncertainty of the fading factor. Furthermore, there will also be fading during the irradiation period itself, i.e. during $t_{i r r}$. If $t_{c a l}$ is of the order of $t_{i r r}$ this should be accounted for in evaluation of the uncertainty in $D_{x}$, for example (approximately) by adding $t_{i r r} / 2$ or $t_{i r} / \ln 2$ to the delay time. It is more correct, however, to make $t_{c a l}>>t_{i r r}$.

It should be noted that, in general, the fading function that is appropriate to the materials being studied should be determined independently and the uncertainty analysis appropriate to that expression should then be evaluated and used. As an example, in case of human teeth as well as integrated circuits from mobile phones, the fading curves were better fitted by a bi-exponential decay function ${ }^{100,102}$. In contrast to electronic components, sensitive dosemeter materials with comparatively slow or no fading, such as household salt $(\mathrm{NaCl})^{101,105}$ and quartz extracted from building materials ${ }^{106-108}$. The application of retrospective luminescence dosimetry in areas affected by fallout from the Semipalatinsk nuclear test site: an evaluation of potential. Health Phys 87(6):625-664), may have a substantial background signal if shielded from light during the time of storage before irradiation. The detection limit in such materials is related to the magnitude and the uncertainty in the background signal. The background absorbed dose in for example household salt may vary depending on how the salt was manufactured $^{101}$, in what package it was kept, and how 
and where this package was stored ${ }^{105}$. For quartz extracted from bricks, the background dose depends on the concentration of natural radionuclides in brick, plaster and soil in front of the building and on the age of the bricks (same references as above). In these cases, when the dose is in the region of the background dose, the variance in the background dose will predominate over the uncertainty in the dose measurement itself. However, for higher doses, in the region of several 100 mGy or above, the uncertainty in background dose will have a lesser impact.

Luminescence signals as recorded by photon counting hardware are in essence a binomial signal, which is supposed to approximate the Poissondistribution when sufficient counts are registered. In OSL, a background is usually subtracted from the measured signal, determined from a certain part of the OSL decay curve (often the last seconds of the measured signal). The background can be a combination of hard-to-bleach components and instrumental background and as such can be overdispersed. Detailed approaches for the calculation of uncertainty for the net OSL count in such a case can be found in the literature, e.g. ${ }^{109}$.

If several calibration doses are used in order to verify the dose-response curve, or several delay times are used to verify the fading curve, a number of different methods are applied. These include proprietary codes or spreadsheets, software for luminescence data processing (e.g. Analyst ${ }^{110}$ ), or dedicated curve fitting packages (e.g. Sigmaplot ${ }^{111}$, Origin $^{112}$ ). The equations chosen to approximate dose responses are commonly linear, saturating exponential (sublinear), or exponential/quadratic (superlinear) in form $^{113}$.

If a sample is divided into several aliquots to assess $D_{X}$, the quantities $I_{X}$ and $I_{c a l}$ could be calculated as the average of the luminescent signals of the different aliquots and their uncertainty as the weighted standard error of the mean. However, to avoid additional uncertainties due to the different aliquot sensitivities, in practice, a dose is usually measured for each aliquot individually and the obtained distribution in aliquot doses further analysed to obtain a best estimate and uncertainty for $D_{X}$.

A variety of approaches has been developed for obtaining central measures from non-perfect data in this case ${ }^{28,114,115}$. Means and maximum likelihood estimates such as the weighted mean (weighted to inverse variance) are associated with well-defined uncertainty estimates ${ }^{21}$, which are obtained by propagation of uncertainties through calculation (internal error) or by evaluation of dispersion in the observed results (external error). Dispersion in observations is commonly observed to be greater than that predicted by propagation of uncertainties through the calculation of the central estimate, leading to the term "over-dispersion". This can relate to experimental variables that are undefined or not included in the calculation, and where signal levels are low. It may also relate to the assumptions underlying the calculations themselves. The combination of data in GUM based approaches assumes a Gaussian approximation of the Poisson distribution.

\section{MONTE CARLO MODELLING TO SUPPORT UNCERTAINTY CALCULATIONS}

A key aim of the EURADOS WG 10 uncertainties task is to further promote the powerful Monte Carlo (MC) techniques for uncertainty estimation. Thus, in addition to the above, we present the following review of use of MC methods within uncertainty estimation. With the availability of high-power computational facilities, numerical simulations have become increasingly practical and popular for analysis of physical or biological systems. One method of numerical simulation that has widespread application in dosimetry, as well as in countless other physical and biological sciences, is the Monte Carlo method.

MC modelling can be used to aid and analyse uncertainty propagation, where the goal is to determine how random variation, lack of knowledge, or error affects the sensitivity, performance, or reliability of the system that is being modelled. However, this inevitably comes at a cost: the MC method is itself prone to uncertainty, and can therefore also be an additional source of error. The current section of this paper focuses on two applications of the Monte Carlo method that are relevant to retrospective dosimetry. The first application discusses the use of Monte Carlo programs created specifically for uncertainty propagation analysis, giving an illustrative example of the technique. The second application concerns the MC transport of ionising radiation through matter, which is a common technique used to model retrospective dosimetry systems. In each case, the role that MC plays in both increasing and decreasing a user's understanding of uncertainty is discussed.

\section{Uncertainty propagation with Monte Carlo}

Monte Carlo Simulation (MCS) provides a practical alternative to the GUM modelling approach. Indeed, the GUM method has limitations, especially in the case in which the model is characterised by a nonlinear function and the approximation of a Taylor's series expansion up to first-order terms for error propagation is not good enough. Furthermore, the uncertainty distributions may be non-Gaussian and is not always 


\section{AINSBURYET AL}

possible to propagate uncertainties using the GUM approach ${ }^{21,116-120}$. The use of MCS for the evaluation of measurement uncertainty is presented by the "GUM Supplement" $"$. MCS may be applied to estimate the combined effects of uncertainty propagation through a physical system that comprises a number of individual components, each of which possesses outcomes and uncertainties expressed by independent probability functions. Many authors report applications of MCS for determination of their measurement uncertainties: Couto et al. ${ }^{116}$, for example, recommended its use for complex problems that could not be solved by the GUM method. Whereas GUM calculations are purely theoretical, MC analyses aim to perform a large series of simulated experiments, with estimates of uncertainties then derived by considering the distributions of their results. In most simple cases, the theoretical GUM results can be compared and tested experimentally against the MC ones. In more complicated situations where the GUM approach would be difficult or unfeasible, MC simulations may still easily provide reliable results. Moreover, whereas the GUM modelling approach may require advanced mathematical skills for many of its procedures, the MCS method can be applied more easily using readily available spreadsheet software, such as Microsoft ${ }^{(R)}$ Excel $^{(R)}$ or Libreoffice Calc: complex uncertainty calculations can hence be accomplished by standard spreadsheet applications rather than by technically demanding mathematical procedures ${ }^{119,120}$.

\section{The MCS method for assessing uncertainty} propagation

$\mathrm{MC}$ analyses require the definition of a measurement model (with the corresponding functional relationship) that describes the measurement process in terms of the inputs to the measurand and the assessment of the types of distribution that apply to the various input uncertainties. The aim of the MC analysis is then to obtain properties of the measurand quantity, $Y$, such as expectations, variances and covariances, and coverage regions, by calculating an approximate numerical representation of the distribution function $G_{Y}$ for $Y$.

Suppose that $Y$ is function of various independent variables $X_{i}$, i.e. $Y=f(\underline{\boldsymbol{X}})=f\left(X_{1}, \ldots, X_{N}\right)$ with $i$ from 1 to $N$; for the present discussion, the $X_{i}$ are assumed to be continuous parameters, but similar techniques can be used for discrete variables. For each input variable $X_{i}$ the corresponding probability density function (PDF), $P\left(X_{i}\right)$, describing its likely values is assumed to be known. A value for $Y$ may therefore be drawn by sampling the $N$ input quantities $X_{i}$ from their respective PDFs. In practice, this sampling procedure is typically achieved computationally using pseudo-random numbers that are generated algorithmically according to a uniform distribution between 0 and 1 , and then suitably "transformed" to obtain the prescribed probability distribution. One such transformation makes use of the cumulative distribution function (CDF), $C\left(X_{i}\right)$, corresponding to a given $P\left(X_{i}\right)$, which is a monotonically increasing, normalizable function with a range constrained between 0 and 1; the result from the MC sampling of the uniform distribution may be identified with a value within the range of this $\mathrm{CDF}$, which then maps uniquely to a specific outcome $X_{i}$. The commonest distributions, $P\left(X_{i}\right)$, used in uncertainty calculations are Gaussian, rectangular, triangular, $t$, exponential, gamma and multivariate Gaussian; it is possible to sample fairly from each of these distributions by using a uniform distribution between 0 and 1 that is randomly sampled by Monte Carlo methods.

Sampling once from each of the $N$ PDFs, $P\left(X_{i}\right)$, corresponding to the $N$ independent input quantities $X_{i}$ provides one value for the measurand, which may be labelled $Y_{1}$, by using the expression of the $f(\underline{X})$ function. Clearly, the value of $Y_{1}$ will depend on the specific outcomes that were obtained during the $N$ random samplings, and repeating the process is likely to yield a different estimate, i.e. $Y_{2}$. If the MCS is repeated $M$ times, requiring $M \times N$ samplings overall, a distribution, $G_{Y}$, of $M$ values for the measurand are generated, i.e. $\left\{Y_{1}, Y_{2}, \ldots, Y_{M}\right\}$. This process is repeated a sufficiently large number of times (i.e. $M$ is very large) in order to have significant statistics, i.e. until it may be assumed that the generated distribution $G_{Y}$ provides a reasonable estimate of the likely distribution of the true measurand, $Y$. Since the input values are randomly drawn from the predefined probability distributions associated with each of the input variables, the information regarding these PDFs will be included implicitly in the distribution of the $Y$ variable, and this allows for the propagation of distributions.

Once the representation $G_{Y}$ of the distribution function for $Y$ has been derived, it is possible to extract from it values for the mean and standard deviation associated with $Y$ as well as the other moments of the distribution function. Moreover, the distribution of output data can be plotted and additional information can be extracted from that graph, such as the coverage interval of the measurand for a stipulated coverage probability, $p$, even when the PDF of the measurand has significant asymmetry. The possibility of graphical representation of the distribution of the measurand through the MCS procedure allows for the individuation of possible asymmetry or deviation from Gaussian shape. This graphical representation favours the determination of a coverage interval corresponding to a stipulated coverage probability.

From the above discussion, the advantages of $\mathrm{MC}$ simulation with respect to the GUM approach ${ }^{21}$ are 


\section{RETROSPECTIVE DOSIMERTY UNCERTAINTIES}

seen to be manifold. The MC technique involves propagation of distributions and always provides a PDF for the output quantity that is consistent with the PDFs of the various inputs, whereas the GUM modelling approach is not able to explicitly determine a PDF for the output quantity. Also, in the case in which the input quantities may themselves depend on other quantities, including corrections and correction factors for type B errors, MCS is able to calculate the combined standard uncertainty of the measurand, even if the functional relationships are complex or difficult to deal with analytically. Similarly, if two inputs are correlated via a bivariate distribution, $\mathrm{MC}$ analysis can provide a joint simulation if the input PDFs are defined in such a way to include the correlation coefficient. Additionally, the MCS procedure intrinsically accounts for any nonlinearity in the functional relationship, whereas GUM does not; in general, more accurate estimates of uncertainties for non-linear models are therefore achieved through MC calculations.

\section{Application of the MCS method for uncertainty propagation}

In order to show how the MC method is used to evaluate measurement uncertainties, an example application for electron paramagnetic resonance (EPR) retrospective dosimetry is reported here. Consider a plot of EPR signal as a function of absorbed dose, and assume that the data may be fitted with a calibration curve characterised by a quadratic trend. This behaviour is common for samples that present a background signal that is of the same shape and overlaps the signal induced by irradiation; in these cases the exposure to ionising radiation produces an increase of EPR intensity of these signals. An example of such a calibration curve is shown in Figure 3.

$<$ Figure 3 here $>$

Figure 3: Example of quadratic calibration curve of EPR signal $v s$. absorbed dose.

The expression for the fitting function in this case is of the general form:

$$
S=a+b \times D+c \times D^{2}
$$

where $S$ is the EPR signal, $D$ is the absorbed dose and $a, b$ and $c$ are the fitting parameters of the calibration curve, which in this example have the values $a=10.4 \pm 0.2, b=1.527 \pm 0.011$ and $c=$ $0.409 \pm 0.005$.

In EPR retrospective dosimetry, the general approach is to reconstruct the absorbed dose $D_{r}$ deposited during an exposure from a measurement of the induced signal, with corrections applied to account for fading and other measurement conditions. Suppose that, as per equation (7) $s$ is the signal measured from a sample, and that the corresponding standard deviation $\sigma_{E D}$ is calculated by considering the various contributions from the standard deviations associated with the fading correction $\left(\sigma_{F}\right)$, the sample preparation process $\left(\sigma_{S}\right)$, the EPR measurement $\left(\sigma_{E}\right)$, and from the numerical treatment of spectra $\left(\sigma_{T}\right)$. For the present example, suppose that $s \pm \sigma_{\mathrm{ED}}$ have the values $25.5 \pm$ 0.7 . The reconstructed dose can be calculated by inverting Equation 23, i.e.:

$$
D=\frac{-b+\sqrt{b^{2}-4 a c+4 c S}}{2 c}
$$

As can be seen, equation (24) contains a fraction and a square root term. The calculation of the standard deviation, $\sigma_{D}$, of the reconstructed dose following the GUM modelling approach is therefore not straightforward because, to take into account the uncertainties of the calibration curve parameters, the corresponding partial derivatives of the factors in equation (24) should be calculated. In fact, under the simplifying hypothesis that the covariances between the various fitting coefficients are negligible, this uncertainty becomes analogous to equation (8):

$$
\sqrt{\left(\frac{d D}{d a}\right)^{2} \sigma_{a+}^{2}\left(\frac{d D}{d b}\right)^{2} \sigma_{b}^{2}+\left(\frac{d D}{d c}\right)^{2} \sigma_{c}^{2}+\left(\frac{d D}{d s}\right)^{2} \sigma_{s}^{2}}
$$

After the calculation of the partial derivatives and the substitution of the above-mentioned values, this approach can be shown to provide the result $D_{r}=$ $4.49 \pm 0.14$ Gy.

On the other hand, this calculation can be performed by MCS following an easy procedure with common spreadsheet software. An example of this MC analysis is reported in Figure 4. In Figure 4, the values in the A, $\mathbf{B}, \mathbf{C}$ and $\mathbf{D}$ columns are realizations of the $s$ measurement and the $a, b$ and $c$ parameters, respectively. All of these quantities are assumed to be distributed normally around their respective average values with their respective standard deviations, which are stated at the top of Figure 4. Based on these distributions, trial values are drawn for each of the input variables ( $a, b, c$ and $s$ ), and the corresponding value of the dose $D$ (column $\mathbf{H}$ ) is then calculated using equation (24). In this example spreadsheet, the values were calculated by using a combination of the NORMINV (for calculating Gaussian-distributed values) and RAND (for generating pseudorandom values) functions, according to the procedure described in the literature ${ }^{120}$. Figure 4 lists the results from ten such applications of this process; for the complete 


\section{AINSBURYET AL}

analysis, a total of $10^{6}$ trials were performed. A histogram of the dose values obtained from these $10^{6}$ trials is reported in Figure 5 binned in 0.02 Gy increments, with the probability (y-axis) derived from a normalization of their respective populations.

\section{$<$ Figure 4 here $>$}

Figure 4: Example of a Monte Carlo simulation for estimating the uncertainty of an EPR measurement, performed using a spreadsheet program.

$<$ Figure 5 here $>$

Figure 5: Histogram of dose values obtained by means of Monte Carlo simulations.

The mean value and the standard deviation of the results are readily calculated from the histogram (Figure 5), and can be shown to be equal to 4.49 Gy and $0.14 \mathrm{~Gy}$, respectively, in this example. These values are consistent with those obtained by the GUM approach. However, as mentioned previously an advantage of the Monte Carlo analysis is that it also provides the PDF for the output quantity, which is dependent on the PDFs of the various inputs. Other moments of the distribution can also be obtained from this PDF, such as the skewness and the kurtosis. These are found to be -0.0588 and 3.0093 respectively for the data in the current example (Figure 5), which are similar to the values of 0 (skewness) and 3 (kurtosis) for a Gaussian distribution, as expected since the PDFs of the input variables were all assumed to be Gaussianlike.

Another application of the Monte Carlo technique is the uncertainty analysis for TL measurements on display glass of mobile phones. The absorbed dose measurement is influenced in this case by the presence of an intrinsic background signal and signal fading ${ }^{103}$. The intrinsic background signal can be reduced by etching the glass sample in concentrated HF before measurement but not completely eliminated ${ }^{104}$. In both cases (etched or unetched), the distribution of intrinsic background doses could be shown to approximately follow a log-normal distribution. From the measured dose $D$, along with its estimated uncertainty $\sigma_{D}$, the corrected unknown absorbed dose $D_{\text {corr }}$ is then calculated from the expression:

$$
D_{\text {corr }}=\frac{D-D_{B G}}{f},
$$

with $D_{B G}$ being the median of the intrinsic background dose distribution and $f$ the fading factor. Analysis of the signal fading of 17 different glass samples for different storage times indicated that the variability (standard deviation) in $f$ is approximately independent of the value of $f$ itself, therefore a constant value for $\sigma_{f}$ is assumed.

Since the calculation of uncertainty for the corrected dose involves the combination of Gaussian and nonsymmetrically distributed parameters, the GUM methodology is not directly applicable whereas with the MCS the simulated distribution of possible corrected dose values is easily obtainable, allowing the immediate assessment of the median and the 95\% confidence interval. An example for two unetched glass samples from mobile phones is shown in Fig. 5. For the sample with the lower dose the uncertainty in the intrinsic background dose dominates, leading to a distribution skewed to the left whereas for the sample with the higher dose, the uncertainty in the fading dominates, leading to a distribution skewed to the right.

$<$ Figure 6here $>$

Figure 6: Histogram of dose values for two display glass samples of irradiated mobile phones (Samsung Galaxy Y S5360). Nominal doses were $0.6 \mathrm{~Gy}$ and $1.5 \mathrm{~Gy}$, reconstructed doses with $95 \%$ CI, 0.59 [0.18-0.83] Gy and 1.6 [1.3 -2.2] Gy, respectively.

\section{Radiation transport modelling with Monte Carlo}

There are a number of Monte Carlo radiation transport codes currently available, examples including the EGSnrc $^{121}$, FLUKA ${ }^{122}$, GEANT ${ }^{123}$, MCNP $^{124}$, PENELOPE $^{125}$ and PHITS ${ }^{126}$ families of software. These codes are described as "general purpose": they are intended, in principle, to be able to model the passage of any type of ionising radiation from any type of source through any arrangement of matter that might be required by their users, providing output data on parameters such as energy depositions and fluences at any location of interest in the geometry. Accordingly, these codes have widespread application in retrospective dosimetry ${ }^{127-133}$, where computer models of the dosimetry system in question may be created and interrogated to understand or improve its performance, limitations and uncertainties. Despite these successes, however, the techniques are not without drawback: although they may be a valuable tool in evaluating and handling uncertainty, they may also be a source of this.

\section{Statistical uncertainties with MC modelling}

It is relatively easy for the users of general purpose $\mathrm{MC}$ codes to reduce the statistical uncertainties on their results. Essentially, these procedures typically rely on increasing the number of scored histories in the regions of interest within the geometry. The most elementary such method is simply to instruct the program to simulate the histories of a greater number of particles, 


\section{RETROSPECTIVE DOSIMERTY UNCERTAINTIES}

though inevitably this is achieved at the expense of an increased CPU time. More sophisticated techniques of variation reduction can also often be implemented, such as biasing source directions, forcing particular interactions to occur, or artificially splitting individual particle histories into multiplicities for example, with scores then having to be weighted accordingly to ensure fairness and fidelity of results. Using these techniques, and coupled with the power of modern computers (especially cluster-based platforms), it is therefore not uncommon for simulated results to be associated with very small statistical uncertainties, sometimes a fraction of a per cent. But anecdotally, at least within the experience of the authors of this current article, such values are often then quoted in the scientific literature as the primary or only uncertainty that is provided with a particular Monte Carlo result. This is misleading, however, because it neglects the type B uncertainties that are also inevitably associated with the modelling, which tend to be much harder to quantify and may be substantially larger in magnitude. Paraphrasing, with Monte Carlo modelling it is often easy to derive highly precise results, but this does not necessarily mean that they are accurate; arguably, the important difference between these two qualities is not always granted enough weight.

\section{Known type B "systematic" uncertainties}

The MC radiation transport method simulates the passage of particles through a user-defined configuration of matter. Accordingly, the accuracy with which the computational model reflects physical reality will dictate the accuracy of its results. Clearly, then, there are a number of factors that could introduce significant uncertainty into the modelling. These might be classified into two broad types: uncertainties inherent in the Monte Carlo software; and uncertainties associated with the user-defined model itself.

Type B uncertainties within the Monte Carlo software incorporate factors such as uncertainties in the underlying physics upon which it relies. These uncertainties include, for example, limitations and inaccuracies of the interaction models that might be in use, including any energy-dependencies that they might have. Although many of these uncertainties may be known in principle, or could be derived from the various references that describe the origins of the physical data and models underpinning the general purpose codes, their magnitudes may not be readily apparent to "casual" users of the software, with their combined effects even harder to quantify. Their contributions to the overall uncertainty budgets arising from the use of Monte Carlo modelling in retrospective dosimetry are therefore highly context dependent, and difficult to numerate in general.
Type B uncertainties that originate from the users of codes reflect the inevitable inability of these users to construct a perfect model of the physical system. This failure might be because of factors that can only be known with limited resolution, and can hence only be input to the MC program with limited accuracy. To give an illustrative example, in the modelling of resistors in mobile phones for fortuitous dosimetry ${ }^{132}$, the absorbed doses received by the target will depend strongly on accurate knowledge of the material compositions and densities of the aluminium oxide substrate, the high- $Z$ contact electrodes adjacent to it, the circuit board to which it is attached, and the screen, case, battery and other features that surround it, as well as on all of their relative locations in 3D space; the estimates of each of these physical parameters will be subject to a significant measurement uncertainty, and this is translated into an unavoidable inaccuracy of the MC model (and hence its results).

Some type B uncertainties may be mitigated by performing sensitivity analyses with the model. In fact, investigating the likely effects of such sensitivities in the physical world might be the primary motivation for developing the Monte Carlo model in the first instance. For example, the impact on dosimetry of the measurement uncertainty on the density of a given object in the real world may be estimated by perturbing the density of that object in the model by an amount deemed equivalent to that uncertainty, and then repeating the simulation; comparison of the perturbed and unperturbed results provides an estimate of the effects of that density uncertainty. Similarly, by varying the concentration of crucial elements, the same approach may be used to estimate the impact of uncertainty regarding the material composition ${ }^{128}$. The MC method is thus seen to be a quick and effective means of quantifying the effects of a given uncertainty in a physical system.

The above univariate sensitivity analysis may be generalised to account for error propagation and the overall uncertainty budget. Specifically, uncertainty propagation analyses can be achieved by repeating the sensitivity analysis for all parameters within the physical model that are associated with a significant type B uncertainty. In fact, this procedure may be applied to assess the impacts of both the physical uncertainties (i.e. the measurement uncertainties) and the code-specific ones; this latter assessment might be achieved by rerunning the simulation using different simulation parameters, for example choosing alternative cross-section databases or interaction models. Overall, the procedure therefore leads to distributions of perturbed data around a mean, which may be interrogated by standard techniques to obtain a handle on the overall quality of the quoted result, and 


\section{AINSBURYET AL}

hence on the robustness of its predictions about the performance of the physical system being modelled.

\section{Unknown type B "systematic" uncertainties}

In addition to applying Monte Carlo techniques to estimate the effects of known type B uncertainties in a physical dosimetry system, such as uncertainties on the precise material composition of a dosemeter, it is also possible to use them to estimate the effects of unknown type B uncertainties. Unknown type B uncertainties include those factors that will affect the results recorded by a retrospective dosemeter, but are a consequence of ignorance of the values of key parameters rather than any imprecision in the estimates of them; these could include uncertainties resulting from incomplete knowledge of the nature of the exposure conditions of the dosemeter, for instance, or missing data on the characteristics of the source term. An example of this might be a situation in which it acknowledged that an energy-dependent correction to the response of a dosemeter needs to be made, but when the energy of the radiation source to which it was exposed is not known. In such cases, it might be appropriate to use Monte Carlo techniques to model the responses of the dosemeter to a range of plausible sources with different energies, with the subsequent variation in the results then used to provide a handle on the maximum error that is likely to be caused by the ignorance of the true energy of the physical exposure.

As an illustrative case study of the application of $\mathrm{MC}$ in handling this type of uncertainty, consider the use of mobile phones as emergency retrospective dosemeters. Although phones, or more specifically their display screens or resistors, possess many of the features considered advantageous to act as reliable fortuitous dosemeters, for them to be useful it is mandatory to relate the absorbed doses they record in an exposure to the concurrent doses deposited in their owners. This can be achieved by Monte Carlo modelling of the phones located at various positions on an anthropomorphic phantom, exposing the configuration to various fields, and then comparing the doses deposited in the phones and phantom to generate a set of exposure- and location-dependent conversion factors; for some locations and exposures, phone and body absorbed doses may differ by a factor of $\sim 20^{129}$. In the real world, however, the precise location of the phone relative to the body during an unplanned exposure may not be known in hindsight, at least not to those performing the dosimetry. Moreover the precise exposure conditions, and orientation of the individual relative to the source, are also unlikely to be recorded. Accordingly, this ignorance introduces a significant unknown type $B$ uncertainty into the conversion of phone doses to body doses. But, this ignorance may be managed by the use of mean conversion factors that are averaged over the datasets of all of the parameters that are unknown. For instance, if the exposure geometry and radiation source were known with some degree of confidence, this averaging might just be over the conversion factor datasets generated by the Monte Carlo model for the different phone positions; but if only the source were known, then the averaging would be over the datasets for all phone positions and all exposure geometries. Use of these mean conversion factors is then associated with conservative uncertainties identified as the maximum over- and under-responses that are expected to arise from their application. These extrema may be taken from the envelope function of the conversion factors that were summed-over in the averaging process, and quantify the worst-case errors in the dosimetry that might be anticipated in adopting this conversion process due to the unknown type B uncertainty in the exposure conditions. This is an important separate topic for further consideration.

\section{BRIEF DISCUSSION AND CONCLUSIONS}

In this paper, the current state of the art in terms of uncertainty analysis techniques for biological dosimetry (with the DCA as the most well developed example) and physical retrospective dosimetry has been reviewed, with a particular emphasis on the potential for increased use of the more sophisticated Bayesian and Monte Carlo modelling methodologies to support uncertainty characterization.

To survey the current situation, a questionnaire was compiled and sent to all members of EURADOS WG10 on retrospective dosimetry. The questionnaire was designed to gather information on current experience on uncertainties estimation but also to assess the possible needs in terms of training or courses. From the 28 laboratories who responded, $72 \%$ currently use physical retrospective dosimetry techniques (EPR, TL and OSL), 19\% biological techniques (micronuclei, dicentrics, FISH, and $\gamma$-H2AX and $8 \%$ others techniques (UV-vis spectroscopy, neutron activation, etc.). $56 \%$ of the responders use only the classical GUM approach, 13\% the approach described in the IAEA manual for cytogenetics, about $18 \%$ Monte Carlo method and about $9 \%$ uses a partial Bayesian approach. None of the responders used a formal Bayesian approach. It is interesting to note that $56 \%$ of responders use software to calculate the uncertainties (35\% in house-developed software, $41 \%$ commercial software and $24 \%$ freely available software). $64 \%$ of responders are satisfied with their method, but would be interested in improving it and/or to evaluate and compare other approach such Bayesian or MC methods, whereas $24 \%$ are aware of the 
weakness of their approach or think they may use an inappropriate one.

So, in general the situation looks positive for the most well-established assays, with practical, accepted analysis techniques in place (chiefly based on GUM) which likely support good overall absorbed dose and uncertainty estimates. The exception is for the more complex exposure scenarios ${ }^{19}$, which are known to introduce additional uncertainties which should ideally be characterised on a case-by-case basis. The tools to do this are in place (for instance in the $\mathrm{GUM}^{21}$ ) but, in practice, are not often applied for biological dose estimation, and to date physical retrospective dosimetry techniques have only really been used for a set of "standard" scenarios. Thus there is still work to do.

The next steps in development of uncertainty analysis techniques across the field of retrospective dosimetry will be to look at standardization of techniques - i.e. to evaluate which of the methods detailed in this work give the most accurate representation of uncertainty in various different exposure scenarios, including the more difficult cases. In addition, as discussed, uncertainty analysis is a complex field in itself and thus an "expert" level of knowledge is required. For example, the application of GUM can be very complex and long, especially when the different terms of uncertainties are correlated (cf. calculation of covariance terms). In many circumstances GUM also requires approximation, and the situation is further complicated when the mathematical model that fits the relation between input data (measurand) and output data (dose) is anything other than linear. The estimation of each of the uncertainty term may need a large amount of work (including experimental work). In light of this, MC or Bayesian approaches would be particularly efficient for retrospective dosimetry application. However, to date, only a small number of EURADOS retrospective dosimetry Group members use these methods, and further work is required. For example, for GUM the algebraic benefits of using the Gaussian approximation should be balanced against its potential divergence from the "true" uncertainty of the observations. However, the way in which the uncertainty is characterised at each stage in a Monte Carlo calculation should be appropriate to the observations and/or should allow for uncertainty in its own assignment ${ }^{28}$, and the extra analytical power provided by the use of Bayesian priors requires that their presence and form be carefully justified in order to limit the potential for mistakes ${ }^{26,31,134}$. In addition, it is worth noting that beside retrospective dosimetry, EPR has long been used for metrology with for instance alanine and recently with tartrates and formats ${ }^{135,136}$. In this field comparatively large amount of effort has ensured that the uncertainty analysis supports highly accurate absorbed dose determinations ${ }^{137}$. However, these principles are equally useful for retrospective dosimetry even if the intrinsic uncertainties are larger and thus the accuracy normally is lower in this case.

It will thus be very important for researchers active in these fields to ensure that new methods are disseminated and that new and existing colleagues access appropriate training. This is something that EURADOS WG10 will continue to support going forward.

\section{FUNDING}

This work was supported by partly supported by the European Radiation Dosimetry Group (EURADOS; WG10 and by the National Institute for Health Research Health Protection Research Unit (NIHR HPRU) in Chemical \& Radiation Threats \& Hazards at Newcastle University in partnership with Public Health England (PHE). The views expressed are those of the author(s) and not necessarily those of the NIHR, the Department of Health or Public Health England.

\section{ACKNOWLEDGMENTS}

The authors would like to express their thanks to the EURADOS WG10 members who supported this work, in particular Octavia Monteiro Gil of the Centro de Ciencias e Tecnologias Nucleares, Instituto Superior Tecnico, Portugal, and Seongjae Jang of the Korea Institute of Radiological and Medical Sciences.

\section{REFERENCES}

1. International Atomic Energy Agency EPRBiodosimetry series. Cytogenetic Dosimetry: Applications in preparedness for and response to radiation emergencies. (2011).

2. Oestreicher, U. et al. RENEB intercomparisons applying the conventional Dicentric Chromosome Assay (DCA). Int. J. Radiat. Biol. 93, 20-29 (2017).

3. Depudyt, J. et al. RENEB intercomparison exercises analyzing micronuclei (Cytokinesis-block

Micronucleus Assay). Int. J. Radiat. Biol. 93, 36-47 (2017).

4. Barquinero, J. F. et al. RENEB biodosimetry intercomparison analyzing translocations by FISH.

Int. J. Radiat. Biol. 93, 30-35 (2017).

5. Terzoudi, G. I. et al. Dose assessment intercomparisons within the RENEB network using $\mathrm{G}_{0}$-lymphocyte prematurely condensed 
chromosomes (PCC assay). Int. J. Radiat. Biol. 93, 48-57 (2017).

6. Moquet, J. et al. The second gamma-H2AX assay inter-comparison exercise carried out in the framework of the European biodosimetry network (RENEB). Int. J. Radiat. Biol. 93, 58-64 (2017).

7. Trompier, F. et al. Overview of physical dosimetry methods for triage application integrated in the new European network RENEB. Int. J. Radiat. Biol. 93, 65-74 (2017).

8. Trompier, F. et al. EPR dosimetry for actual and suspected overexposures during radiotherapy treatments in Poland. Radiat. Meas. 42, 1025-1028 (2007).

9. Degteva, M. O. et al. Analysis of EPR and FISH studies of radiation doses in persons who lived in the upper reaches of the Techa River. Radiat. Environ. Biophys. 54, 433-44 (2015).

10. Trompier, F. et al. EPR Retrospective Dosimetry with Fingernails. Health Phys. 106, 798-805 (2014).

11. Sholom, S. \& McKeever, S. W. S. Emergency EPR dosimetry technique using vacuum-stored dry nails. Radiat. Meas. 88, 41-47 (2016).

12. Marciniak, A. \& Ciesielski, B. EPR dosimetry in nails-A review. Appl. Spectrosc. Rev. 51, 73-92 (2016).

13. Fattibene, P. et al. EPR dosimetry intercomparison using smart phone touch screen glass. Radiat.

Environ. Biophys. 53, 311-20 (2014).

14. Bailiff, I. K., Sholom, S. \& McKeever, S. W. S. Retrospective and emergency dosimetry in response to radiological incidents and nuclear mass-casualty events: A review. Radiat. Meas. 94, 83-139 (2016).

15. Jaworska, A. et al. Operational guidance for radiation emergency response organisations in Europe for using biodosimetric tools developed in eu multibiodose project. Radiat. Prot. Dosimetry 164, 165-169 (2015).

16. Kulka, U. et al. Realising the European network of biodosimetry: Reneb-status quo. Radiat. Prot.

Dosimetry 164, 42-45 (2015).

17. Kulka, U. et al. RENEB - Running the European Network of biological dosimetry and physical retrospective dosimetry. Int. J. Radiat. Biol. 93, 214 (2017).

18. Helton, J. C. in 207-228 (Springer Berlin Heidelberg, 2008). doi:10.1007/978-3-540-7736279
19.

Vinnikov, V. A., Ainsbury, E. A., Lloyd, D. C., Maznyk, N. A. \& Rothkamm, K. Difficult cases for chromosomal dosimetry: Statistical considerations. Radiat. Meas. 46, 1004-1008 (2011).

20. Ainsbury, E. et al. Integration of new biological and physical retrospective dosimetry methods into EU emergency response plans - joint RENEB and EURADOS inter-laboratory comparisons. Int. J. Radiat. Biol. 93, 99-109 (2017).

21. ISO/IEC. Uncertainty of measurement - Guide to the expression of uncertainty in measurement (GUM:1995). ISO/IEC Guide 98-3:2008 2008, (2008).

22. International Standard Organization. ISO/IEC 17025 General requirements for the competence of testing and calibration laboratories. Int. Stand. 2005, 1-36 (2005).

23. JCGM 100. JCGM 100:2008 - Evaluation of measurement data - Guide to the expression of uncertainty in measurement. Int. Organ. Stand. Geneva ISBN 50, 134 (2008).

24. ISO 5725-1:1994 Accuracy (trueness and precision) of measurement methods and results - Part 1: General principles and definitions.

25. ISO 13528:2015 Statistical methods for use in proficiency testing by interlaboratory comparison. (2015).

26. Sivia, D. S. \& Skilling, J. (John). Data analysis : a Bayesian tutorial. (Oxford University Press, 2006).

27. Marrale, M. et al. Assessing the Impact of Copy Number Variants on miRNA Genes in Autism by Monte Carlo Simulation. PLoS One 9, e90947 (2014).

28. Sivia, D. S., Burbidge, C., Roberts, R. G. \& Bailey, R. M. A Bayesian Approach to the Evaluation of Equivalent Doses in Sediment Mixtures for Luminescence Dating. in AIP Conference Proceedings 735, 305-311 (AIP, 2004).

29. Thomsen, K. J., Murray, A. S. \& Bøtter-Jensen, L. Sources of variability in OSL dose measurements using single grains of quartz. Radiat. Meas. 39, 4761 (2005).

30. Higueras, M., Puig, P., Ainsbury, E. A., Vinnikov, V. A. \& Rothkamm, K. A new Bayesian model applied to cytogenetic partial body irradiation estimation. Radiat. Prot. Dosimetry 168, 330-6 (2016).

31. Ramsey, C. B. Deposition models for chronological 
records. Quat. Sci. Rev. 27, 42-60 (2008).

32. Sigurdson, A. J. et al. International study of factors affecting human chromosome translocations. Mutat. Res. Toxicol. Environ. Mutagen. 652, 112-121 (2008).

33. McNamee, J. P., Flegal, F. N., Greene, H. B., Marro, L. \& Wilkins, R. C. Validation of the cytokinesis-block micronucleus (CBMN) assay for use as a triage biological dosimetry tool. Radiat. Prot. Dosimetry 135, 232-42 (2009).

34. Flegal, F. N., Devantier, Y., McNamee, J. P. \& Wilkins, R. C. Quickscan dicentric chromosome analysis for radiation biodosimetry. Health Phys. 98, 276-81 (2010).

35. ISO 19238:2014 Radiological protection -

Performance criteria for service laboratories performing biological dosimetry by cytogenetics. (2014).

36. ISO 21243:2008 Radiation protection Performance criteria for laboratories performing cytogenetic triage for assessment of mass casualties in radiological or nuclear emergencies - General principles and application to dicentric assay. (2008).

37. Vral, A., Fenech, M. \& Thierens, H. The micronucleus assay as a biological dosimeter of in vivo ionising radiation exposure. Mutagenesis 26, 11-7 (2011).

38. ISO 17099:2014 Radiological protection -Performance criteria for laboratories using the cytokinesis block micronucleus (CBMN) assay in peripheral blood lymphocytes for biological dosimetry.

39. Higueras, M. et al. A new inverse regression model applied to radiation biodosimetry. Proc. R. Soc. A 471: 20140, (2015).

40. Ainsbury, E. A. \& Barquinero, J. F. Biodosimetric tools for a fast triage of people accidentally exposed to ionizing radiation. Statistical and computational aspects. Ann. Ist. Super. Sanita 45, 307-312 (2009).

41. Fenech, M. The lymphocyte cytokinesis-block micronucleus cytome assay and its application in radiation biodosimetry. Health Phys. 98, 234-43 (2010).

42. Pernot, E. et al. Ionizing radiation biomarkers for potential use in epidemiological studies. Mutat. Res. Mutat. Res. 751, 258-286 (2012).

43. Ainsbury, E. A. et al. What radiation dose does the fish translocation assay measure in cases of incorporated radionuclides for the Southern Urals populations? Radiat. Prot. Dosimetry 159, 26-33 (2014).

44. Barnard, S. et al. The first gamma-H2AX biodosimetry intercomparison exercise of the developing european biodosimetry network RENEB. Radiat. Prot. Dosimetry 164, 265-270 (2015).

45. Rothkamm, K., Krüger, I., Thompson, L. H. \& Löbrich, M. Pathways of DNA double-strand break repair during the mammalian cell cycle. Mol. Cell. Biol. 23, 5706-15 (2003).

46. Rothkamm, K. \& Horn, S. gamma-H2AX as protein biomarker for radiation exposure. Ann. dell'Istituto Super. di sanità 45, 265-71 (2009).

47. Horn, S. \& Rothkamm, K. Candidate protein biomarkers as rapid indicators of radiation exposure. Radiat. Meas. 46, 903-906 (2011).

48. Valdiglesias, V., Laffon, B., Pásaro, E. \& Méndez, J. Evaluation of Okadaic Acid-Induced Genotoxicity in Human Cells Using the Micronucleus Test and $\gamma \mathrm{H} 2 \mathrm{AX}$ Analysis. J. Toxicol. Environ. Heal. Part A 74, 980-992 (2011).

49. Wojcik, A. et al. Multidisciplinary Biodosimetric Tools for a Large-scale Radiological Emergency the MULTIBIODOSE Project. 3, 19-23 (2014).

50. Rübe, C. E. et al. DNA repair in the context of chromatin: New molecular insights by the nanoscale detection of DNA repair complexes using transmission electron microscopy. DNA Repair (Amst). 10, 427-437 (2011).

51. Löbrich, M. et al. In vivo formation and repair of DNA double-strand breaks after computed tomography examinations. Proc. Natl. Acad. Sci. U. S. A. 102, 8984-9 (2005).

52. Rothkamm, K., Balroop, S., Shekhdar, J., Fernie, P. \& Goh, V. Leukocyte DNA damage after multidetector row CT: a quantitative biomarker of lowlevel radiation exposure. Radiology 242, 244-51 (2007).

53. Sedelnikova, O. A. et al. Delayed kinetics of DNA double-strand break processing in normal and pathological aging. Aging Cell 7, 89-100 (2008).

54. Joyce, E. F. et al. Drosophila ATM and ATR have distinct activities in the regulation of meiotic DNA damage and repair. J. Cell Biol. 195, 359-367 (2011). 
55. Ricceri, F. et al. Involvement of MRE11A and XPA gene polymorphisms in the modulation of DNA double-strand break repair activity: A genotypephenotype correlation study. DNA Repair (Amst). 10, 1044-1050 (2011).

56. Valdiglesias, V., Giunta, S., Fenech, M., Neri, M. \& Bonassi, S. $\gamma \mathrm{H} 2 \mathrm{AX}$ as a marker of DNA double strand breaks and genomic instability in human population studies. Mutat. Res. Mutat. Res. 753, 24 40 (2013).

57. Horn, S., Barnard, S. \& Rothkamm, K. GammaH2AX-Based Dose Estimation for Whole and Partial Body Radiation Exposure. PLoS One 6, e25113 (2011).

58. Abragam, A. \& Bleaney, B. Electron Paramagnetic Resonance of Transition Ions $\mid A$. Abragam and B. Bleaney | 9780199651528 | Oxford University Press Canada. (ClearendonPress, 1970).

59. Weil, A. J. \& Bolton, J. in Electron Paramagnetic Resonance: Elementary Theory and Practical Applications (ed. Wiley) (2006). doi:10.1002/0470084987

60. Poole, C. P. Electron Spin Resonance: A Comprehensive Treatise on Experimental Techniques. (Dover Publications, 1997).

61. Trompier, F. et al. Radiation-induced signals analysed by EPR spectrometry applied to fortuitous dosimetry. Ann. dell'Istituto Super. di sanità 45, 287-96 (2009).

62. Israelsson, A., Gustafsson, H. \& Lund, E. Dose response of xylitol and sorbitol for EPR retrospective dosimetry with applications to chewing gum. Radiat. Prot. Dosimetry 154, 133-41 (2013).

63. Trompier, F., Bassinet, C. \& Clairand, I. RADIATION ACCIDENT DOSIMETRY ON PLASTICS BY EPR SPECTROMETRY. Health Phys. 98, 388-394 (2010).

64. Sholom, S. \& Chumak, V. EPR EMERGENCY DOSIMETRY WITH PLASTIC COMPONENTS OF PERSONAL GOODS. Health Phys. 98, 395399 (2010).

65. Kamenopoulou, V., Barthe, J., Hickman, C. \& Portal, G. Accidental Gamma Irradiation Dosimetry Using Clothing. Radiat. Prot. Dosimetry 17, 185188 (1986).

66. Barthe, J., Kamenopoulou, V., Cattoire, B. \& Portal, G. Dose evaluation from textile fibers: a post- determination of initial ESR signal. Int. J. Radiat. Appl. Instrumentation. Part A. Appl. Radiat. Isot. 40, 1029-1033 (1989).

67. McKeever, S. W. S. et al. Numerical solutions to the rate equations governing the simultaneous release of electrons and holes during thermoluminescence and isothermal decay. Phys. Rev. B 32, 3835-3843 (1985).

68. Woda, C. et al. Radiation-induced damage analysed by luminescence methods in retrospective dosimetry and emergency response. Ann. Ist. Super. Sanita 45, 297-306 (2009).

69. Bassinet, C. et al. Retrospective radiation dosimetry using OSL of electronic components: Results of an inter-laboratory comparison. Radiat. Meas. 71, 475479 (2014).

70. Vinnikov, V. A., Ainsbury, E. A., Maznyk, N. A., Lloyd, D. C. \& Rothkamm, K. Limitations associated with analysis of cytogenetic data for biological dosimetry. Radiat. Res. 174, 403-14 (2010).

71. Savage, J. R. K. \& Papworth, D. G. Constructing a 2B Calibration Curve for Retrospective Dose Reconstruction. Radiat. Prot. Dosimetry 88, 69-76 (2000).

72. Merkle, W. Statistical methods in regression and calibration analysis of chromosome aberration data. Radiat. Environ. Biophys. 21, 217-233 (1983).

73. Szłuińska, M., Edwards, A. A., Lloyd, D. C. \& Health Protection Agency (Great Britain). Radiation Protection Division. Statistical methods for biological dosimetry. (Health Protection Agency, Radiation Protection Division, 2005).

74. Ainsbury, E. A., Vinnikov, V. A., Maznyk, N. A., Lloyd, D. C. \& Rothkamm, K. A comparison of six statistical distributions for analysis of chromosome aberration data for radiation biodosimetry. Radiat. Prot. Dosimetry 155, 253-267 (2013).

75. Ainsbury, E. A. et al. Uncertainty of fast biological radiation dose assessment for emergency response scenarios. Int. J. Radiat. Biol. 93, 127-135 (2017).

76. Schenker, N. \& Gentleman, J. F. On Judging the Significance of Differences by Examining the Overlap Between Confidence Intervals. http://dx.doi.org/10.1198/000313001317097960 (2012).

77. Austin, P. \& HUX, J. A brief note on overlapping confidence intervals. J Vasc Surg 36, 194-195 
(2002).

78. Deperas, J. et al. CABAS: a freely available PC program for fitting calibration curves in chromosome aberration dosimetry. Radiat. Prot. Dosimetry 124, 115-23 (2007).

79. Ainsbury, E. A. \& Lloyd, D. C. Dose estimation software for radiation biodosimetry. Health Phys. 98, 290-295 (2010).

80. Sasaki, M. S. Chromosomal biodosimetry by unfolding a mixed Poisson distribution: a generalized model. Int. J. Radiat. Biol. 79, 83-97 (2003).

81. Ainsbury, E. A. et al. CytoBayesJ: Software tools for Bayesian analysis of cytogenetic radiation dosimetry data. Mutat. Res. - Genet. Toxicol. Environ. Mutagen. 756, 184-191 (2013).

82. Brame, R. S. \& Groer, P. G. Bayesian analysis of overdispersed chromosome aberration data with the negative binomial model. Radiat. Prot. Dosimetry 102, 115-9 (2002).

83. DiGiorgio, M. \& Zaretzky, A. Biological dosimetry - A Bayesian approach for presenting uncertainty on biological dose estimates. Annals of 'II Encuen- tro de Docentes e Investigadores de Estadstica en Psicologa (2011).

84. Groer, P. G. \& De Pereira, C. A. B. in Probability and Bayesian Statistics 225-232 (Springer US, 1987). doi:10.1007/978-1-4613-1885-9 23

85. Edwards, A. A. \& Lloyd, D. C. in The Early Effects of Radiation on DNA 385-396 (Springer Berlin Heidelberg, 1991). doi:10.1007/978-3-642-751486_40

86. Moriña, D., Higueras, M., Puig, P., Ainsbury, E. A. \& Rothkamm, $\mathrm{K} .<\mathrm{tt}>$ radir $</ \mathrm{tt}>$ package: an $<\mathrm{tt}>\mathrm{R}</ \mathrm{tt}>$ implementation for cytogenetic biodosimetry dose estimation. J. Radiol. Prot. 35, 557-569 (2015).

87. IAEA et al. Use of electron paramagnetic resonance dosimetry with tooth enamel for retrospective dose assessment. Vienna IAEA 57 (2002).

88. Fattibene, P., La Civita, S., De Coste, V. \& Onori, $\mathrm{S}$. Analysis of sources of uncertainty of tooth enamel EPR signal amplitude. Radiat. Meas. 43, 827-830 (2008).

89. Nagy, V. Accuracy considerations in EPR dosimetry. Appl. Radiat. Isot. 52, 1039-1050 (2000).

90. ISO/ASTM 51607:2013 - Practice for use of the
alanine-EPR dosimetry system. (2013).

91. Fattibene, P. \& Callens, F. EPR dosimetry with tooth enamel: a review. Appl. Radiat. Isot. 68, 2033-2116 (2010).

92. Fattibene, P., Duckworth, T. L. \& Desrosiers, M. F. Critical evaluation of the sugar-EPR dosimetry system. Appl. Radiat. Isot. 47, 1375-1379 (1996).

93. Wilcox, D. E. et al. DOSIMETRY BASED ON EPR SPECTRAL ANALYSIS OF FINGERNAIL CLIPPINGS. Health Phys. 98, 309-317 (2010).

94. Anton, M. et al. Uncertainties in alanine/ESR dosimetry at the Physikalisch-Technische Bundesanstalt. Phys. Med. Biol. 51, 5419-5440 (2006).

95. Antonovic, L., Gustafsson, H., Carlsson, G. A. \& Carlsson Tedgren, A. Evaluation of a lithium formate EPR dosimetry system for dose measurements around 192Ir brachytherapy sources. Med. Phys. 36, 2236-47 (2009).

96. Wieser, A. et al. Assessment of performance parameters for EPR dosimetry with tooth enamel. Radiat. Meas. 43, 731-736 (2008).

97. Currie, L. A. Nomenclature in evaluation of analytical methods including detection and quantification capabilities: (IUPAC Recommendations 1995). Anal. Chim. Acta 391, 105-126 (1999).

98. Currie, L. A. Detection and quantification limits: basic concepts, international harmonization, and outstanding ('low-level') issues. Appl. Radiat. Isot. 61, 145-149 (2004).

99. Fattibene, P. et al. The 4th International comparison on EPR dosimetry with tooth enamel, part 1: report on the results. Radiat. Meas. 46, 765-771 (2011).

100. Sholom, S., Dewitt, R., Simon, S. L., Bouville, A. \& McKeever, S. W. S. Emergency Dose Estimation Using Optically Stimulated Luminescence from Human Tooth Enamel. Radiat. Meas. 46, 778-782 (2011).

101. Bernhardsson, C., Christiansson, M., Mattsson, S. \& Rääf, C. L. Household salt as a retrospective dosemeter using optically stimulated luminescence. Radiat. Environ. Biophys. 48, 21-28 (2009).

102. Sholom, S. \& McKeever, S. W. S. Integrated circuits from mobile phones as possible emergency OSL/TL dosimeters. Radiat. Prot. Dosimetry (2015). doi:10.1093/rpd/ncv446

103. Discher, M. \& Woda, C. Thermoluminescence of 
glass display from mobile phones for retrospective and accident dosimetry. Radiat. Meas. 53-54, 12-21 (2013).

104. Discher, M., Woda, C. \& Fiedler, I. Improvement of dose determination using glass display of mobile phones for accident dosimetry. Radiat. Meas. 56, 240-243 (2013).

105. Christiansson, M., Bernhardsson, C., GeberBergstrand, T., Mattsson, S. \& Rääf, C. L. Household salt for retrospective dose assessments using OSL: signal integrity and its dependence on containment, sample collection, and signal readout. Radiat. Environ. Biophys. 53, 559-69 (2014).

106. Woda, C. et al. Luminescence dosimetry in a contaminated settlement of the Techa River valley, Southern Urals, Russia. Radiat. Meas. 46, 277-285 (2011).

107. Woda, C. et al. Evaluation of external exposures of the population of Ozyorsk, Russia, with luminescence measurements of bricks. Radiat. Environ. Biophys. 48, 405-417 (2009).

108. Bailiff, I. K. et al. The application of retrospective luminescence dosimetry in areas affected by fallout from the semipalatinsk nuclear test site: an evaluation of potential. Health Phys. 87, 625-41 (2004).

109. Galbraith, R. F. A further note on the variance of a background - corrected OSL count. Anc. TL 32, 1-3 (2014).

110. Duller, G. A. T. \& T., G. A. The Analyst software package for luminescence data: overview and recent improvements. Anc. TL 33, 35-42 (2015).

111. Sigmaplot. (2017). Available at: http://www.sigmaplot.co.uk/products/peakfit/peakfit .php. (Accessed: 22nd February 2017)

112. Origin. (2017). Available at: http://www.originlab.com/index.aspx?go=Solutions/ Applications/Spectroscopy. (Accessed: 22nd February 2017)

113. Burbidge, C. I. A broadly applicable function for describing luminescence dose response. J. Appl. Phys. 118, 044904 (2015).

114. Committee, A. M. Robust statistics: a method of coping with outliers. AMC Tech. Br. 2 (2001).

115. Galbraith, R. F., Roberts, R. G., Laslett, G. M., Yoshida, H. \& Olley, J. M. Optical dating of single and multiple grains of quartz from jinmium rock sheltern, northern Australia: Part I, experimental design and statistical models. Archaeometry 41, 339-364 (1999).

116. Couto, P. R. G., Damasceno, J. C. \& Oliveira, S. P. Monte Carlo Simulations Applied to Uncertainty in Measurement. Theory Appl. Monte Carlo

Simulations 27-51 (2013).

117. Ángeles Herrador, M. \& González, A. G. Evaluation of measurement uncertainty in analytical assays by means of Monte-Carlo simulation. Talanta 64, 415422 (2004).

118. Lepek, A. A computer program for a general case evaluation of the expanded uncertainty. Accredit.

Qual. Assur. 8, 296-299 (2003).

119. Chew, G. \& Walczyk, T. A Monte Carlo approach for estimating measurement uncertainty using standard spreadsheet software. Anal. Bioanal. Chem. 402, 2463-9 (2012).

120. Farrance, I. \& Frenkel, R. Uncertainty in measurement: a review of monte carlo simulation using microsoft excel for the calculation of uncertainties through functional relationships, including uncertainties in empirically derived constants. Clin. Biochem. Rev. 35, 37-61 (2014).

121. Kawrakow, I. \& Rogers, D. The EGSnrc code system: Monte Carlo simulation of electron and photon transport. NRCC Rep. PIRS-701 (2000).

122. Ferrari, A., Sala, P. R., Fasso, A. \& Ranft, J. FLUKA: a multi-particle transport code. (2005).

123. Agostinelli, S. et al. Geant4 - a simulation toolkit. Nucl. Instruments Methods Phys. Res. Sect. A Accel. Spectrometers, Detect. Assoc. Equip. 506, 250-303 (2003).

124. Pelowitz, D. B. E. MCNP6TM users manual Version 1.0. LA-CP-13-00634. (2013).

125. Salvat, F., Fernandez-Varea, J. M. \& Sempau, J. Penelope-2006: A code system for Monte Carlo simulation of electron and photon transport workshop proceedings, Barcelona, Spain, 4-7 July 2006 - nea6222-penelope.pdf. (2006).

126. Niita, K., Matsuda, N., Iwamoto, Y., Iwase, H. \& Sato, T. PHITS : Particle and Heavy Ion Transport code System, Version 2 . 23. JAEA-Data/Code (2010).

127. Discher, M. Lumineszenzuntersuchungen an körpernah getragenen Gegenständen für die Notfalldosimetrie. (München, Technische Universität, 2015).

128. Discher, M., Hiller, M. \& Woda, C. MCNP 
simulations of a glass display used in a mobile phone as an accident dosimeter. Radiat. Meas. 75, 21-28 (2015).

129. Eakins, J. S. \& Kouroukla, E. Luminescence-based retrospective dosimetry using $\mathrm{A} 12 \mathrm{O} 3$ from mobile phones: a simulation approach to determine the effects of position. J. Radiol. Prot. 35, 343-81 (2015).

130. Gómez-Ros, J. M., Pröhl, G., Ulanovsky, A. \& Lis, M. Uncertainties of internal dose assessment for animals and plants due to non-homogeneously distributed radionuclides. J. Environ. Radioact. 99, 1449-1455 (2008).

131. Hervé, M. L., Clairand, I., Trompier, F., Tikunov, D. \& Bottollier-Depois, J. F. Relation between organ and whole body doses and local doses measured by ESR for standard and realistic neutron and photon external overexposures. Radiat. Prot. Dosimetry 125, 355-60 (2007).

132. KOUROUKLA, E. Luminescence dosimetry with ceramic materials for application to radiological emergencies and other incidents. (2015).

133. Ulanovsky, A., Pröhl, G. \& Gómez-Ros, J. M. Methods for calculating dose conversion coefficients for terrestrial and aquatic biota. $J$. Environ. Radioact. 99, 1440-1448 (2008).

134. Chipman, H., George, E. \& MuCulloch, R. The practical implementation of Bayesian model selection. IMS Lect. Notes - Monogr. Ser. 38, (2001).

135. Lund, E. et al. Formates and dithionates: sensitive EPR-dosimeter materials for radiation therapy. Appl. Radiat. Isot. 62, 317-324 (2005).

136. Marrale, M. et al. Neutron ESR dosimetry through ammonium tartrate with low Gd content. Radiat.

Prot. Dosimetry 159, 233-6 (2014).

137. Bergstrand, E. S., Hole, E. O. \& Sagstuen, E. A Simple Method for Estimating Dose Uncertainty in ESR/Alanine Dosimetry. Appl. Radiat. Isot. 7, 845854 (1998). 